\title{
An Input-Representing Interneuron Regulates Spike Timing and Thereby Phase Switching in a Motor Network
}

\author{
Kosei Sasaki, Jian Jing, Michael R. Due, and Klaudiusz R. Weiss \\ Department of Neuroscience, Mount Sinai School of Medicine, New York, New York 10029
}

\begin{abstract}
Despite the importance of spike-timing regulation in network functioning, little is known about this regulation at the cellular level. In the Aplysia feeding network, we show that interneuron B65 regulates the timing of the spike initiation of phase-switch neurons B64 and cerebral-buccal interneuron-5/6 (CBI-5/6), and thereby determines the identity of the neuron that acts as a protraction terminator. Previous work showed that $\mathrm{B} 64$ begins to fire before the end of protraction phase and terminates protraction in CBI-2-elicited ingestive, but not in CBI-2-elicited egestive programs, thus indicating that the spike timing and phase-switching function of B64 depend on the type of the central pattern generator (CPG)-elicited response rather than on the input used to activate the CPG. Here, we find that CBI-5/6 is a protraction terminator in egestive programs elicited by the esophageal nerve (EN), but not by CBI-2, thus indicating that, in contrast to B64, the spike timing and protraction-terminating function of CBI-5/6 depends on the input to the CPG rather than the response type. Interestingly, B65 activity also depends on the input in that B65 is highly active in EN-elicited programs, but not in CBI-2-elicited programs independent of whether the programs are ingestive or egestive. Notably, during EN-elicited egestive programs, hyperpolarization of B65 delays the onset of CBI-5/6 firing, whereas in CBI-2-elicited ingestive programs, B65 stimulation simultaneously advances CBI-5/6 firing and delays B64 firing, thereby substituting CBI-5/6 for B64 as the protraction terminator. Thus, we identified a neural mechanism that, in an input-dependent manner, regulates spike timing and thereby the functional role of specific neurons.
\end{abstract}

Key words: Aplysia; central pattern generator; feeding; spike timing; phase transition; motor programs

\section{Introduction}

Spike timing is critical for a variety of neural functions that include pattern generation, plasticity, neuromodulation, and neural coding (Bi and Poo, 1998; Roberts and Bell, 2002; Murphy and Rieke, 2006; Robbe et al., 2006; Sakurai et al., 2006; Cassenaer and Laurent, 2007; Wu et al., 2007). Studies in Aplysia (Wu et al., 2007) have shown that in the feeding central pattern generating (CPG) network, the timing of the spike initiation of interneuron B64 is an important determinant of its ability to terminate the protraction and initiate the retraction phase of motor programs. Specifically, B64 acts as a phase-switch neuron when its firing is initiated before, but not after, the end of protraction. In turn, the onset of B64 firing depends on the state of the network, as the timing of B64 activity depends on whether the network generates ingestive versus egestive motor programs. Thus, whether B64 functions as a protraction terminator does not depend on the input used to trigger these programs. Because B64 is a phaseswitch neuron under some, but not other, circumstances there must exist additional phase-switch neuron(s) whose spike timing relative to B64 is likely to be regulated. Given that spike-timing changes are regulated not only in Aplysia, but also in other systems, and yet the mechanisms of such regulation are poorly un-

Received 0ct. 19, 2007; revised Jan. 13, 2008; accepted Jan. 15, 2008.

This work was supported by the National Institutes of Health Grant MH35564.

Correspondence should be addressed to Dr. Klaudiusz R. Weiss, Department of Neuroscience, Box 1065, Mount Sinai School of Medicine, 1 Gustave Levy Place, New York, NY 10029. E-mail: Klaudiusz.Weiss@mssm.edu. DOI:10.1523/JNEUROSCI.4755-07.2008

Copyright $\odot 2008$ Society for Neuroscience $\quad$ 0270-6474/08/281916-13\$15.00/0 derstood, we sought to examine such mechanisms in Aplysia whose small network greatly simplifies this task.

Previous work demonstrated that similar to B64, the cerebralbuccal interneuron-5/6 (CBI-5/6) (Perrins and Weiss, 1998) begins to fire during the transition from protraction to retraction and can terminate protraction when prematurely activated (Sasaki et al., 2007). These findings led us to hypothesize that CBI-5/6 may be one of the missing phase-switch neurons. Here, we show that CBI-5/6 is not a protraction terminator in ingestive programs elicited by a command-like neuron, CBI-2. Importantly, however, we show that CBI-5/6 functions as a protraction terminator in egestive programs elicited by stimulation of the esophageal nerve (EN), but not in egestive programs elicited by CBI-2 stimulation. Thus, CBI-5/6 functions appear to depend on the input to the CPG, but not the program type elicited by this input, i.e., $\mathrm{CBI}-5 / 6$ is a phase-switch neuron when programs are elicited via EN, but not via CBI-2 stimulation. Additionally, the selection of B64 versus CBI-5/6 as a phase-switch neuron is regulated by an input-representing CPG interneuron, B65 (Proekt et al., 2007). B65 is strongly active during EN-elicited egestive programs, but not during CBI-2-elicited ingestive or egestive programs. B65 advances the firing of CBI-5/6 and delays the firing of $\mathrm{B} 64$ relative to protraction termination. These characteristics allow B65 to regulate the relative spike timing of CBI-5/6 versus B64, thereby altering their phase-switching functions in an inputdependent manner. Thus, our study underscores the importance of those CPG elements that represent inputs rather than those that represent responses/outputs. By regulating the timing of ac- 
A



B1

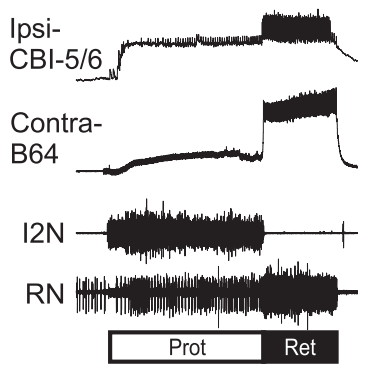

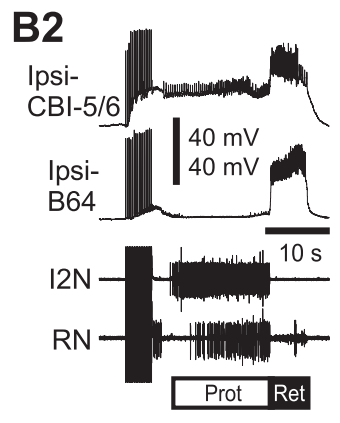

Figure 1. The schematic diagram of the core circuitry that mediates the protraction-retraction sequence of Aplysia feeding motor programs and activity patterns of $\mathrm{CBI}-5 / 6$ and $\mathrm{B} 64$ in different types of programs. $A$, Two inputs, $\mathrm{CBI}-2$ and EN, were used to activate the CPG. A single cycle of the Aplysia feeding motor programs consists of two phases, a radula protraction phase followed by a radula retraction phase. During protraction, Pls drive PMs, which in turn activate protraction muscles. PIs also inhibit Rls and RMs. During retraction, Rls drive RMs, which in turn activate retraction muscles. Rls also inhibit Pls and PMs. Protraction/retraction phase transitions are triggered, at least partly, through slow excitation of RIs by Pls, which, at a delay, overcomes inhibition of Rls by Pls. MNs, Motorneurons. Connections: Open triangle, excitation; filled circle, inhibition; broken line, polysynaptic connection. $\boldsymbol{B}$, Examples of activity patterns of $\mathrm{CBI}-5 / 6$ and $\mathrm{B} 64$ in ingestive motor programs elicited by $\mathrm{CBI}-2$ stimulation (B1) or in egestive motor programs elicited by EN stimulation (B2). Protraction (Prot; open bar) is monitored by activity in the I2N. Retraction (Ret; filled bar) is monitored by robust activities of CBI-5/6 and B64, both of which are active throughout the retraction phase in both types of motor programs. Radula closure activity is monitored on the basis of activity in the RN, which contains the axons of the radula closer motoneurons B8. In the (BI-2-elicited ingestive program (B1), RN activity primarily occurred during the retraction phase; in the EN-elicited egestive program (B2), RN activity primarily occurred during the protraction phase. Each neuron is labeled with a prefix such as ipsilateral (Ipsi-) and contralateral (Contra-) related to the side on which CBI-2 or EN was stimulated. In subsequent figures, neurons are also labeled this way.

tivity of other CPG elements, such neurons may well play an important role in regulating, in an input-dependent manner, the functional configuration of the CPG.

\section{Materials and Methods}

Experiments were performed on Aplysia californica (100-200 g) obtained from Marinus (Long Beach, CA). Animals were anesthetized by injection of an isotonic $\mathrm{MgCl}_{2}$ solution (30-50\% of body weight). The cerebral and buccal ganglia were removed, desheathed, and pinned in a recording chamber $\left(14-17^{\circ} \mathrm{C}\right.$; volume, $\left.\approx 1.1 \mathrm{ml}\right)$ perfused at $0.3-0.35$ $\mathrm{ml} / \mathrm{min}$. The composition of normal artificial seawater (ASW) was (in mм) $460 \mathrm{NaCl}, 10 \mathrm{KCl}, 11 \mathrm{CaCl}_{2}, 55 \mathrm{MgCl}_{2}$, and $10 \mathrm{HEPES}$ at $\mathrm{pH}$ 7.6. The composition of a high-divalent-cation (Hi-Di) ASW was (in mM) 348 $\mathrm{NaCl}, 8 \mathrm{KCl}, 23.4 \mathrm{CaCl}_{2}, 116 \mathrm{MgCl}_{2}$, and 10 HEPES at pH 7.6.

Electrophysiology. Intracellular recordings were made using singlebarrel electrodes (4-10 M $\Omega$ ) filled with $2 \mathrm{M} \mathrm{KAc}$ and $30 \mathrm{~mm} \mathrm{KCl}$. Intracellular signals were acquired using an AxoClamp 2B amplifier (Molecular Devices, Union City, CA) or Getting Model 5A amplifier (Getting Instruments, San Diego, CA). Extracellular signals were acquired from polyethylene suction electrodes using a Differential AC Amplifier (model 1700; A-M Systems, Carlsborg, WA). A Grass Stimulator (model S88; Grass Medical Instruments, Quincy, MA) was used for stimulation.

Neurons were identified as described previously (Rosen et al., 1991; Hurwitz and Susswein, 1996; Kabotyanski et al., 1998; Perrins and Weiss, 1998).

To elicit individual egestive programs, unless otherwise indicated, the EN was stimulated with $5 \mathrm{~Hz}, 2 \mathrm{~ms}$ trains of current pulses for $4 \mathrm{~s}$ (see Fig. $1 \mathrm{B2}$ ). In biasing experiments, EN was stimulated continuously at 1.5-2 $\mathrm{Hz}$ with $2 \mathrm{~ms}$ current pulses, the amplitude of which was adjusted to elicit four to five successive cycles of motor programs. A S48 stimulator (Grass Medical Instruments) was used to generate current pulses that were injected into the extracellular polyethylene suction electrode into which the esophageal nerve had been aspirated.

Motor programs were also elicited via stimulation of CBI-2. To prevent activation of CBI-3 via electrical coupling we did not use a DC predepolarization of CBI-2 (Morgan et al., 2002). Motor programs were elicited by stimulation of CBI-2 $(8-10 \mathrm{~Hz})$. Each current pulse was set to trigger a single action potential. CBI-2 stimulation was manually termi- nated after the protraction phase has ended as determined on the basis of the activity of the I2 nerve ( $22 \mathrm{~N})$.

Statistics. Data were plotted using SigmaPlot 8 (SPSS, Chicago, IL), and statistics were performed in Prism 4 (GraphPad Software, San Diego, CA). Data are presented as mean \pm SEM. Data were analyzed using a repeated one-way ANOVA or Student's $t$ test. Data that showed significant effects in ANOVA were further compared using Bonferroni's multiple-comparison tests. Difference is considered significant when $p<0.05$.

\section{Results}

The multifunctional feeding CPG of Aplysia generates several types of motor programs which can be elicited through activation of external inputs (e.g., CBI-2 and EN) (Fig. 1) (Rosen et al., 1991; Morton and Chiel, 1993b). The major components of these programs are the ones that generate the protraction/retraction and the opening/closing movements of the radula. Independent of the type of output that the CPG generates, the protraction/retraction components occur in a fixed sequence in which the protraction phase precedes the retraction phase (Fig. 1). The protraction phase is initiated when protraction interneurons (PIs) begin to fire because of excitation from inputs such as CBI-2 and EN (Fig. 1A). The PIs drive a number of protraction motoneurons (PMs), and inhibit the retraction interneurons (RIs) and retraction motoneurons (RMs). The RIs, which fire during retraction, inhibit the PIs and a number of PMs. The protraction phase was monitored (Fig. $1 B$, open bar) by the activity in the $\mathrm{I} 2 \mathrm{~N}$, which contains axons of bilateral B31/32s and B61/62s that are active only during the protraction phase and are actively inhibited during the retraction phase (Hurwitz et al., 1994, 1996). Because of this feature, the $\mathrm{I} 2 \mathrm{~N}$ provides an integrated readout of the protraction phase. The retraction phase was monitored (Fig. $1 B$, filled bar) by intracellular recordings from B64 or CBI-5/6, both of which fire throughout the retraction phase of both ingestive and egestive programs. Consistent with other studies in the field (Hurwitz et al., 1996; Nargeot et al., 1997), we operationally defined the time at which the protraction phase ended as the time at which the last spike was recorded in the $\mathrm{I} 2 \mathrm{~N}$.

In contrast to the phase-fixed characteristics of the protraction/retraction sequence, the timing of the radula closing depends on the type of program. Basically, when the radula closure motoneuron B8 fires predominantly during retraction and, thus, helps bring the food into the buccal cavity, the program is considered to be ingestive. When B8 fires predominantly during protraction and, thus, helps in removing a nonedible object from the buccal cavity, the program is considered to be egestive (Church and Lloyd, 1994; Nargeot et al., 1997; Sanchez and Kirk, 2000; Jing and Weiss, 2001; Morgan et al., 2002). Examples of ingestive and egestive motor programs are shown in Figure $1 B$. Figure $1 B 1$ is an example of an ingestive program in which the radula nerve (RN), containing axons of bilateral B8s (Morton and Chiel, 1993a), is active predominantly during retraction. Figure $1 B 2$ is an example of an egestive program in which $\mathrm{RN}$ is active predominantly during protraction. 
Activity of the soma and the axon of CBI$5 / 6$ in ingestive and egestive programs CBI-5/6 is a pair of bilaterally symmetrical interganglionic interneurons: the soma of CBI-5/6 is located in the cerebral ganglion, and its axon projects to the buccal ganglion which contains the feeding CPG (Fig. 2A) (Perrins and Weiss, 1998). Previous studies suggested that the axonal region, but not the somatic region of CBI-5/6 can act as an element of the buccal CPG (Perrins and Weiss, 1998; Sasaki et al., 2007). These studies also suggested that during the retraction phase of motor programs, action potentials of CBI-5/6 may be initiated in the CBI-5/6 axon within the buccal ganglion, and then propagate toward the soma of the cerebral ganglion (Perrins and Weiss, 1998; Sasaki et al., 2007). However, although the indirect evidence supported the above suggestions, direct evidence in the form of intracellular recordings from axons was missing. Given the relevance of this point to the role of CBI-5/6 as an element of the CPG, we sought to determine whether during motor programs the action potentials in CBI-5/6 originate in the buccal or in the cerebral ganglia.

We obtained simultaneous intracellular recordings from the soma and the axon of CBI-5/6. The location of the recording sites is shown in Figure $2 A$. In Figure $2 B 1$, a depolarizing current $(2.0 \mathrm{nA})$ was injected into the soma of CBI-5/6. Orthodromic spikes that were recorded in the axon followed the spikes in the soma and occurred without any previous depolarizing deflection. Similarly, as shown in Figure 2 B2, axon-initiated spikes were elicited by current injection $(2.3 \mathrm{nA})$ into the axon of CBI-5/6. These spikes propagated antidromically toward the soma and preceded one-for-one the antidromic spikes recorded in the soma (Fig. 2B2). The antidromic spikes recorded in the soma were much smaller than the spikes recorded in the axon. This result is consistent with the previous finding that the amplitude of the action potentials recorded in CBI-5/6 soma tends to be $<20 \mathrm{mV}$ (Perrins and Weiss, 1998) (Fig. 2 B2, top). Also, consistent with the idea that the depolarizations recorded in the soma of CBI-5/6 represented antidromic spikes, these depolarizing potentials became even smaller when the soma was hyperpolarized (Fig. 2 B2, bottom). Finally, the mean latency from an axoninitiated spike to an antidromic spike recorded in the soma was $43.2 \pm 1.9 \mathrm{~ms}(n=$ 11), whereas the mean latency from a spike elicited by current injection into the soma to an orthodromic spike recorded in the axon was $27.0 \pm 3.6 \mathrm{~ms}(n=11)$. This dif-
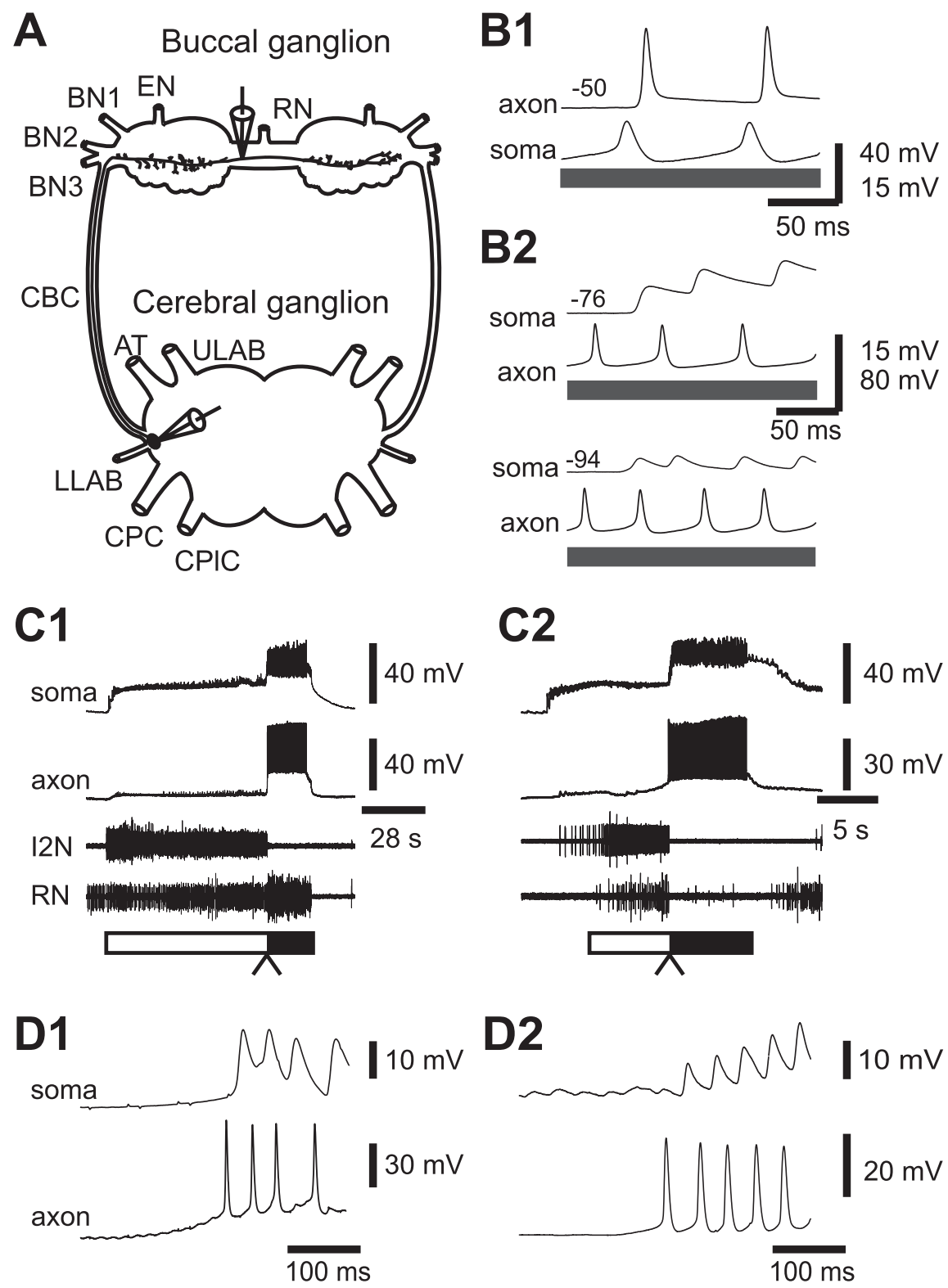

Figure 2. Spike propagation and activity of $\mathrm{CBI}-5 / 6$ soma and axon. $A$, The schematic drawing of CBI-5/6 illustrates the sites at which our recordings were obtained. One electrode was used to impale the soma of $\mathrm{CBI}-5 / 6$ in the cerebral ganglion (dorsal surface). The second electrode was used to impale the axon of $\mathrm{CBI}-5 / 6$ in the buccal ganglion (rostral surface). The distance between the recording sites was $\sim 10 \mathrm{~mm}$. $\boldsymbol{B}$, Simultaneous recordings from the soma and the axon of CBI-5/6. B1, A somatic current injection (gray bar) elicited action potentials in the soma and these were followed by action potentials in the axon. B2, Axon-initiated spikes elicited by current injection were followed one-for-one by antidromic spikes in the soma (top). Hyperpolarization ( $-18 \mathrm{mV}$ ) of (BI-5/6 soma dramatically reduced the amplitude of antidromic spikes (bottom). Note that antidromic spikes in the soma were smaller than the axon-initiated spikes. Membrane potential of $\mathrm{CBI}-5 / 6$ axon or soma is shown at the left (in millivolts). C, CBI-5/6 was recruited in both CBI-2- and EN-elicited programs. Simultaneous recordings from the axon and the soma of $\mathrm{CBI}-5 / 6$ during a $\mathrm{CBI}-2$-elicited ingestive program (C1) and an EN-elicited egestive program (C2). These recordings were obtained from ipsilateral CBI-5/6 to the side on which CBI-2 or EN was stimulated. The protraction and retraction phases of motor programs are marked by open and filled bars, respectively. In CBI-2-elicited programs, RN was predominantly active during retraction, indicating that the program was ingestive, whereas in EN-elicited programs, RN was predominantly active during protraction, indicating that the program was egestive. Note that in both programs, during the protraction phase, $\mathrm{CBI}-5 / 6$ soma received excitatory synaptic inputs, but no spikes were present in the axon. However, during the retraction phase, a barrage of action potentials is present both in the axon and in the soma of $\mathrm{CBI}-5 / 6$. D, Expanded views of the transition between the protraction and the retraction phases ( $\mathbf{C}, \mathbf{C}$, arrowheads) in (BI-2-elicited ingestive programs ( $\mathbf{D} 1$ is from $\mathbf{C 1}$ ) and in EN-elicited egestive programs (D2 is from (2). In both programs, spikes in the axon were followed one-for-one by antidromic spikes in the soma, indicating that when programs are generated, the spikes of $\mathrm{CBI}-5 / 6$ originate in the axonal region and propagate toward the soma. BN, Buccal nerve; $C B C$, cerebral-to-buccal connective; $U L A B$, upper labial nerve; $A T$, anterior tentacular nerve; LLAB, lower labial nerve; CPC, cerebral-to-pedal connective; CPIC, cerebral-to-pleural connective. 


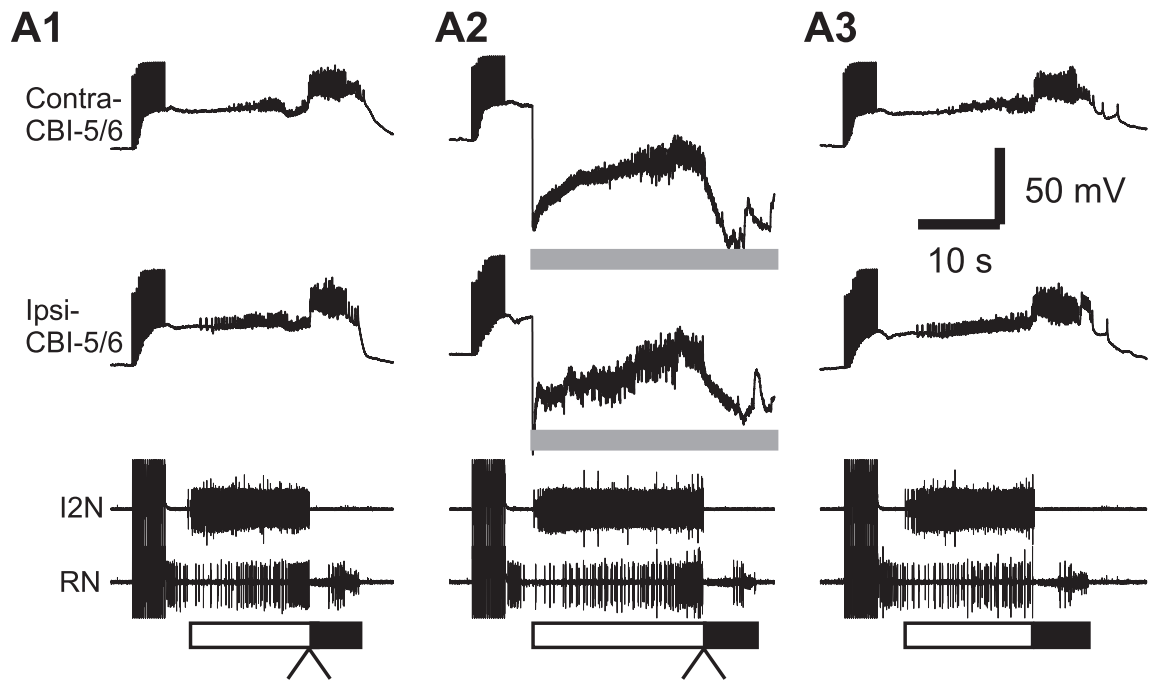

B1

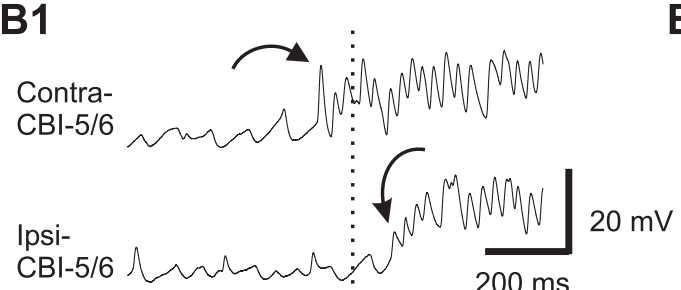

B2

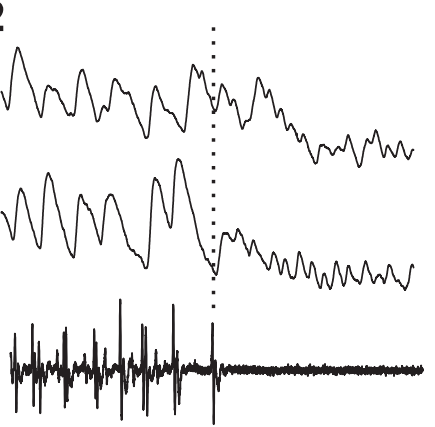

Figure 3. Effect of $\mathrm{CBI}-5 / 6$ hyperpolarization on the duration of the protraction phase in EN-elicited egestive programs. A1, An EN-elicited egestive program in control condition. A2, Bilateral hyperpolarization (gray bars) of ipsilateral CBI-5/6 and contralateral $\mathbf{C B I}-5 / 6$ extended protraction duration. $\boldsymbol{A}$, The program after the hyperpolarization was released. $\boldsymbol{B}$, Expanded views of the transition between the protraction and retraction phases ( $\boldsymbol{A} 1, \boldsymbol{A 2}$, arrowheads) without $\mathrm{CB}$-5/6 hyperpolarization ( $\boldsymbol{B} 1$ is from A1) and with (BI-5/6 hyperpolarization ( $B 2$ is from $\boldsymbol{A 2}$ ). Vertical dashed lines are drawn just above the last spike in I2N, to facilitate the visualization of the difference between the end of the protraction phase and the onset of the antidromic spikes in bilateral CBI-5/6 somata (arrows). Antidromic spikes in one (BI-5/6 appear before the end of I2N activity (B1). Synaptic inputs in CBI-5/6 during the protraction phase were unmasked during hyperpolarization, and the amplitude of antidromic spikes in CBI-5/6 was decreased, making it difficult to distinguish the first antidromic spike (B2).

ference was statistically significant $(p<0.001)$, suggesting that the spike initiation zone, which is responsible for the attenuated spikes recorded in the soma, is located far from the soma. This zone may be located near or even within the buccal ganglion as distally initiated spike would have to traverse a longer distance to reach the soma than to reach the buccal ganglion.

We next examined the activities of both the soma and the axon of CBI-5/6 during the motor programs elicited by stimulation of CBI-2 or EN (Fig. 2C,D). When motor programs are repeatedly elicited by stimulation of CBI-2, the feeding CPG tends to generate ingestive programs in which motoneuronal activity in $\mathrm{RN}$ is higher during the retraction phase than during the protraction phase (Fig. 2C1). However, when EN is repeatedly stimulated, the CPG generates egestive programs in which the level of activity in $\mathrm{RN}$ is higher during the protraction phase than during the retraction phase (Fig. 2C2). In both types of motor programs, CBI-5/6 axon fired spikes during retraction. More importantly, the attenuated antidromic spikes recorded in CBI-5/6 soma were preceded one-for-one by spikes of its own axon (Fig. 2D1,D2), indicating that indeed the action potentials originated in the buccal region of CBI-5/6. The average spike frequencies during the first second, the first four seconds, and for the whole duration of the retraction phase were, respectively, $20.8 \pm 2.2 \mathrm{~Hz}$, $17.9 \pm 1.6 \mathrm{~Hz}$, and $11.1 \pm 1.6 \mathrm{~Hz}(n=4)$ in CBI-2-elicited ingestive programs, and $22.3 \pm 1.8 \mathrm{~Hz}, 18.3 \pm 0.8 \mathrm{~Hz}$, and $14.4 \pm$ $1.7 \mathrm{~Hz}(n=4)$ in EN-elicited egestive programs. There was no significant difference between the average firing frequencies in the ingestive versus egestive programs in any of the three periods for which the firing frequency was measured $(p=0.63, p=$ 0.84 , and $p=0.10$ for the first second, the first four seconds, and the whole duration, respectively). These results show that CBI$5 / 6$ is recruited at high frequency in both CBI-2-elicited ingestive programs and ENelicited egestive programs, and that in both programs spikes recorded in the axon precede those recorded in the soma.

\section{CBI-5/6 functions as a protraction terminator in EN-elicited egestive programs, but not in CBI-2-elicited ingestive programs}

Previous study showed that somatic stimulation of CBI-5/6 early in the protraction phase prematurely terminated the protraction phase and advanced the transition to the retraction phase (Sasaki et al., 2007). Thus, this study indicated that CBI-5/6 could potentially act as a protraction terminator in motor programs. However, it remained unknown whether CBI-5/6 actually acts as a protraction terminator in motor programs, and if so in what types of motor programs. To characterize the potential contribution of CBI-5/6 to protraction termination in different types of motor programs, we bilaterally hyperpolarized CBI-5/6 somata. If CBI-5/6 were to function as a primary protraction terminator, then hyperpolarization of CBI-5/6 could be expected to delay the termination of the protraction phase and thus extend the protraction duration.

We first examined effects of CBI-5/6 hyperpolarization on the protraction duration of EN-elicited egestive programs. Specifically, we hyperpolarized either CBI-5 or CBI-6 on each side of the cerebral ganglion. Ipsilateral CBI-5 and CBI-6 are electrically coupled (Perrins and Weiss, 1998). The hyperpolarization was accomplished by a direct current ( $\sim 20 \mathrm{nA}$ ) injection. Figure 3 illustrates representative recordings obtained in this type of experiment. Recordings in Figure $3 A 2$ were obtained while bilateral CBI-5/6s were hyperpolarized. Recordings in Figure 3, $A 1$ and $A 3$, were obtained respectively before and after the recordings in which CBI-5/6s were hyperpolarized. Hyperpolarization of CBI-5/6 increased the amplitude of the small potentials recorded in CBI-5/6 during protraction (Fig. 3, compare B1, B2). These potentials were previously identified as EPSPs (Perrins and Weiss, 1998; Sasaki et al., 2007). In contrast, the potentials recorded in CBI-5/6 during the retraction phase became smaller as a result of CBI-5/6 hyperpolarization. This is consistent with our data showing that these potentials represent antidromic spikes (Fig. 2).

Despite the fact that hyperpolarization of CBI-5/6 did not appear to block completely the ability of CBI-5/6 to generate spikes, somatic hyperpolarization of CBI-5/6 was effective in ex- 
tending the duration of the protraction phase (Fig. 3A). Because direct stimulation of CBI-5/6 terminates the protraction phase (Sasaki et al., 2007), it is likely that the onset of its activity was delayed by hyperpolarization. Compared with the control groups, the hyperpolarization of CBI$5 / 6$ on average increased the mean duration of protraction by $23.6 \pm 3.2 \%$ $(n=11)$ (see Fig. $6 A$ ). The overall difference between the groups, before, during, and after hyperpolarization of CBI-5/6 was statistically significant (see Fig. $6 A)\left(F_{(2,20)}\right.$ $=40.11, p<0.0001)$. Bonferroni's multiple-comparison tests showed that during hyperpolarization of CBI-5/6, the protraction duration was significantly different from the protraction duration before $(p<0.001)$ and after hyperpolarization $(p<0.001)$. The difference between protraction durations before and after hyperpolarization was not significant ( $p=$ $0.42)$.

The expanded records of the protraction-to-retraction transitions (Fig. $3 B$ ) illustrate the timing of CBI-5/6 firing relative to the end of protraction. In here and in subsequent experiments, the latency of CBI-5/6 firing relative to $\mathrm{I} 2 \mathrm{~N}$ activity is defined as the time between the last spike recorded in $\mathrm{I} 2 \mathrm{~N}$ and the first antidromic spike recorded in CBI-5/6 soma. If the first antidromic spike precedes the last spike in $\mathrm{I} 2 \mathrm{~N}$, we define the latency of CBI-5/6 firing as negative; if the first antidromic spike follows the last spike in $\mathrm{I} 2 \mathrm{~N}$, we define the latency of CBI-5/6 firing as positive. Data analysis of CBI-5/6 firing refers to the CBI$5 / 6$ that was the first one to fire when bilateral CBI-5/6s activities were monitored. In all of the recordings, the ipsilateral and contralateral refer to the side on which CBI-2 or EN was stimulated. The data show that, in the control condition (Fig. 3B1), the latency of antidromic spikes recorded in the soma of one of the bilateral CBI-5/6s was negative relative to the end of $\mathrm{I} 2 \mathrm{~N}$ activity, i.e., the spike in this CBI-5/6 was initiated before the end of I2N activity (Fig. 3B1). The average latency of the antidromic spikes of CBI-5/6 in EN-elicited egestive programs was $-57.1 \pm 11.2 \mathrm{~ms}(n=11)$ (see Fig. $6 E)$. Because it was found previously that current-injection-induced firing of CBI$5 / 6$ early in the protraction phase shortens the duration of this phase (Sasaki et al., 2007) and now we show that CBI-5/6 begins to fire before the end of the protraction phase and that CBI-5/6 hyperpolarization extends the duration of protraction, the combination of these findings support the hypothesis that CBI-5/6 contributes to the termination of protraction and the transition to retraction in EN-elicited egestive programs. Because CBI-5/6 hyperpolarization dramatically reduced the size of the antidromic spikes in programs in which CBI-5/6 was hyperpolarized, we were not able to unequivocally determine the exact time at which the first CBI-5/6 spike was generated during hyperpolarization and, thus, could not determine the exact latency of CBI$5 / 6$ firing relative to the termination of $\mathrm{I} 2 \mathrm{~N}$ activity. Thus, at this time it remains unclear whether, in programs in which we hyper-
A2
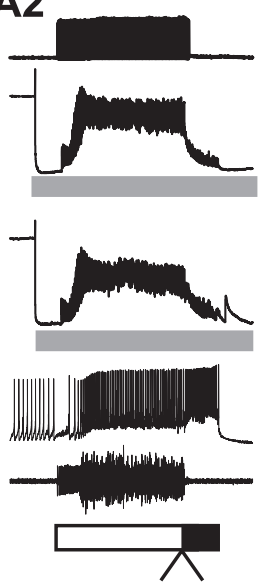

A3

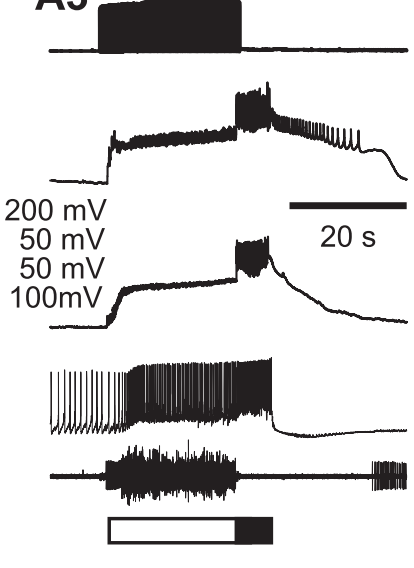

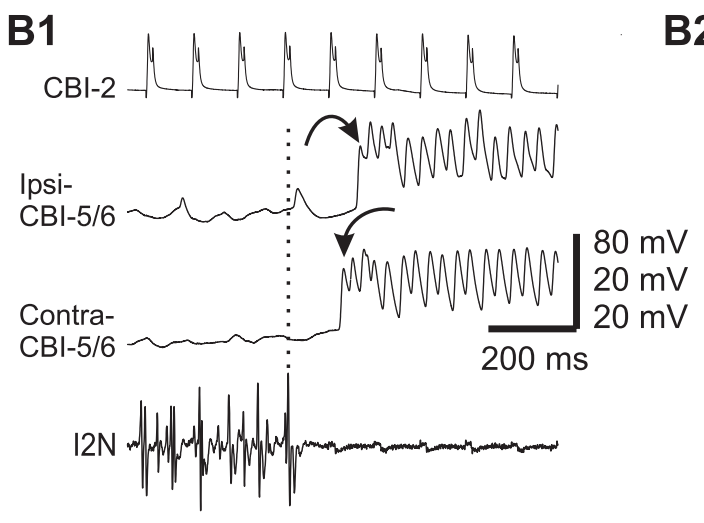

B2
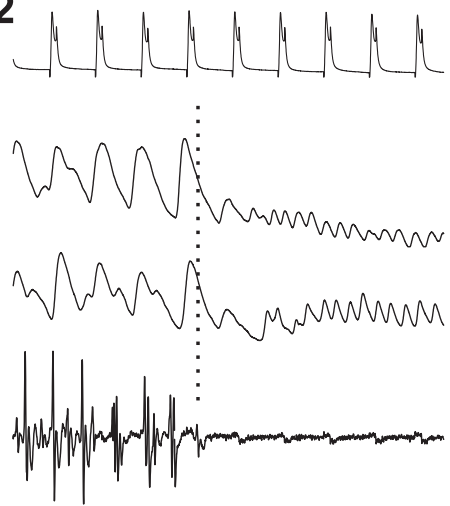

Figure 4. Effect of $\mathrm{CBI}-5 / 6$ hyperpolarization on the duration of the protraction phase in $\mathrm{CBI}-2$-elicited ingestive programs. $\boldsymbol{A 1}$, A CBI-2-elicited ingestive program in control condition. $\boldsymbol{A 2}$, Bilateral hyperpolarization of $\mathrm{CBI}-5 / 6$ had no effect on protraction duration. $\boldsymbol{A}$, The program after the hyperpolarization was released. $\boldsymbol{B}$, Expanded views of the transition between the protraction and retraction phases ( $\boldsymbol{A} \mathbf{1}, \boldsymbol{A} \mathbf{2}$, arrowheads) without $\mathrm{CB}-5 / 6$ hyperpolarization ( $\boldsymbol{B}$ 1 is from $\boldsymbol{A} \mathbf{1}$ ) and with $\mathrm{CBI}-5 / 6$ hyperpolarizaactivity (B1). In these experiments, (BI-2 stimulation was manually terminated after the protraction phase has ended. Note that CBI-2 stimulation continued for a brief period of time after the end of protraction and the beginning of retraction $(\mathbf{B} 1, \mathbf{B 2})$.

polarized $\mathrm{CBI}-5 / 6$, the prolongation of protraction duration allowed another neuron, including the nonhyperpolarized CBI$5 / 6$, to step in and terminate the protraction phase or alternatively whether the hyperpolarized CBI-5/6 still acted as a protraction terminator, albeit with a delay. Independent of which scenario operates, it is clear that CBI-5/6 hyperpolarization delays the termination of the protraction phase and that these findings support the hypothesis that CBI-5/6 may act as a protraction terminator in EN-elicited egestive programs.

We next investigated the effect of CBI-5/6 hyperpolarization in CBI-2-elicited ingestive programs. In contrast to the observations made for EN-elicited egestive programs, the protraction duration of CBI-2-elicited ingestive programs was not affected by CBI-5/6 hyperpolarization (Figs. $4 A, 6 B)\left(F_{(2,12)}=1.02, p=\right.$ $0.39)$. To exclude the possibility that the ineffectiveness of CBI$5 / 6$ hyperpolarization could be caused by variability between preparations, in four of the seven preparations, we elicited programs via both CBI-2 and EN stimulation (see Fig. $6 \mathrm{C}$ ). In these four preparations, CBI-5/6 hyperpolarization extended the protraction duration of EN-elicited egestive programs (see Fig. 6C1) $\left(F_{(2,6)}=11.15, p<0.01\right)$, but not of CBI-2-elicited ingestive programs (see Fig. $6 C 2)\left(F_{(2,6)}=1.87, p=0.23\right)$, suggesting that variability between preparations was not a major factor. Consistent with the lack of effect of CBI-5/6 hyperpolarization on the 

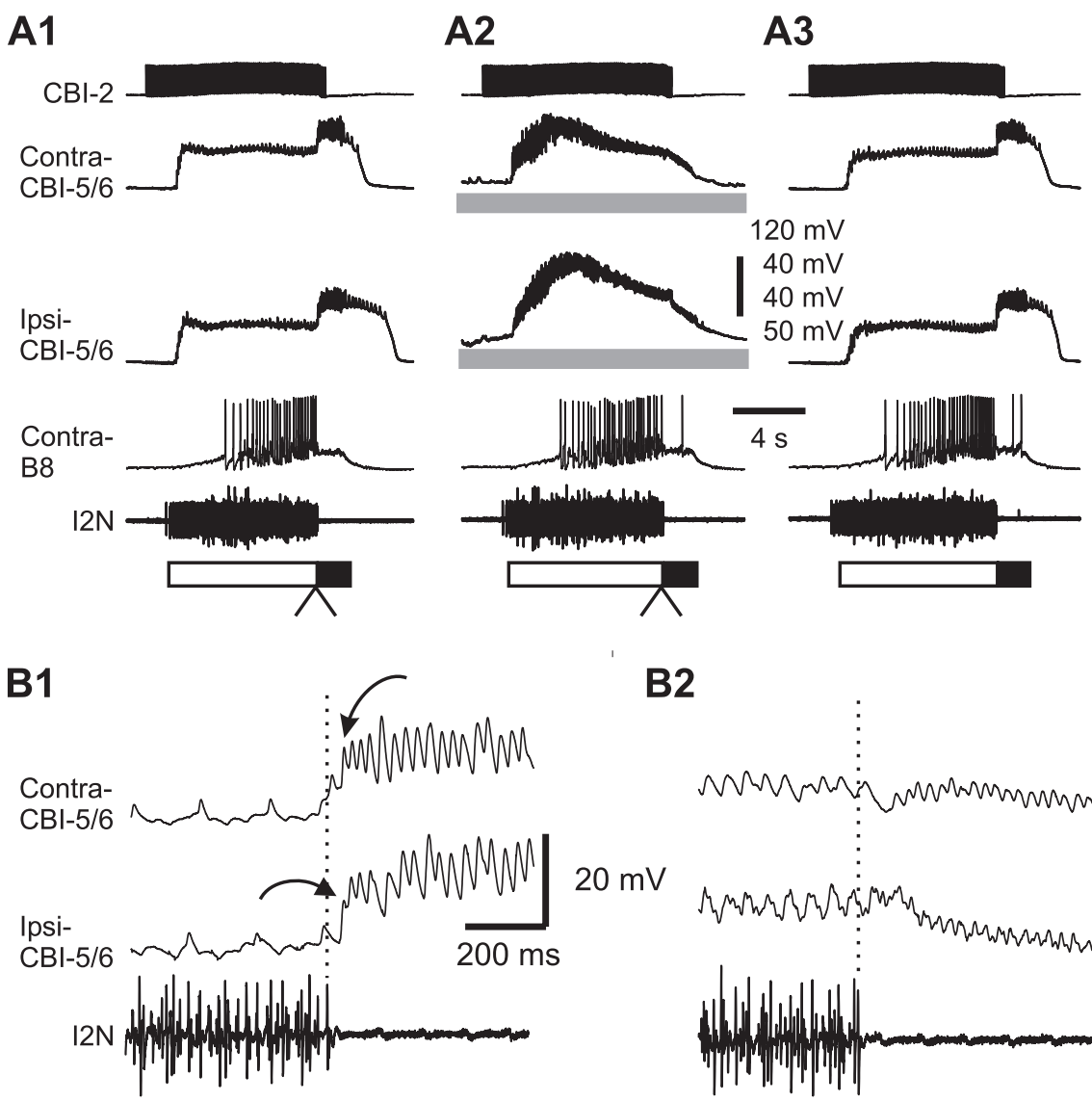

B2

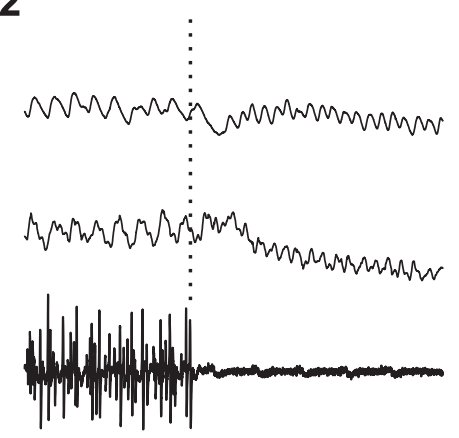

Figure 5. Effect of $\mathrm{CBI}-5 / 6$ hyperpolarization on the duration of the protraction phase in $\mathrm{CBI}-2$-elicited egestive programs. $\boldsymbol{A}$, A CBI-2-elicited egestive program in control condition. Before the programs were elicited by CBI-2 stimulation, four successive programs were evoked by continuous stimulation of EN $(1.5 \mathrm{~Hz})$. Note that, compared with $\mathrm{CBI}$-2-elicited ingestive programs (Figs. $4 A, 8 A$, and $10 A$ ), the high level of $B 8$ activity occurred during the protraction phase. $\boldsymbol{A 2}$, Bilateral hyperpolarization of CBI-5/6 had no effect on protraction duration. $A 3$, The program after the hyperpolarization was released. $B$, Expanded views of the transition between the protraction and retraction phases ( $\boldsymbol{A} 1, \boldsymbol{A 2}$, arrowheads) without $\mathrm{CB}-5 / 6$ hyperpolarization ( $\boldsymbol{B} 1$ is from $\boldsymbol{A 1}$ ) and with (BI-5/6 hyperpolarization (B2 is from $\boldsymbol{A 2}$ ). Note that in $\mathrm{CBI}$-2-elicited egestive programs, antidromic spikes in CBI-5/6 began after the end of I2N activity $(B 1)$.

protraction duration in $\mathrm{CBI}-2$-elicited ingestive programs, we found that in control conditions, antidromic spikes of CBI-5/6 occurred after the end of I2N activity (Fig. 4B1). The mean latency of antidromic spikes of CBI-5/6 in CBI-2-elicited ingestive programs was positive, $80.3 \pm 26 \mathrm{~ms}$ (see Fig. $6 E)(n=7)$, i.e., CBI-5/6 firing was initiated after the protraction phase was already terminated, thus making it unlikely that CBI-5/6 acted as a protraction terminator in CBI-2-elicited ingestive programs.

\section{Input dependence of CBI-5/6 functions: CBI-2-elicited egestive program}

The programs in which we compared the protraction terminating actions of CBI-5/6 differed not only in that they were ingestive versus egestive, but also in that they were elicited by different means. Specifically, ingestive programs were elicited by CBI-2 stimulation, whereas egestive programs were elicited by EN stimulation. It was, therefore, conceivable that it was not the nature of the program that determined whether CBI-5/6 acted as a protraction terminator but that instead it was the input used to elicit the program that determined whether $\mathrm{CBI}-5 / 6$ acted as a protraction terminator. Specifically, if CBI-5/6 actions depended on the nature of programs, they could act as a protraction terminator for egestive programs regardless of how these programs were elicited. Alternatively, if CBI-5/6 actions depended on the input, they could act as a protraction terminator only when EN stimulation was used to elicit the program. We, therefore, sought to dissociate the nature of the programs from the inputs that were used to elicit them.

Previous work showed that in the aftermath of a series of EN stimulations, the state of the buccal CPG is altered (Proekt et al., 2004, 2007). In this new state, CBI-2 stimulation exclusively elicits egestive rather than ingestive programs. We exploited this observation to determine whether CBI- $5 / 6$ acts as a protraction terminator in an input- or responsedependent manner. Specifically, before CBI-2 stimulation, we elicited four to five successive egestive programs via EN stimulation. Stimulation of CBI-2 now consistently produced egestive programs (Fig. 5). Comparisons of EN- and CBI-2-elicited egestive programs showed that, in these programs elicited by two different inputs, there were no statistically significant differences in protraction duration, in B8 firing frequency during protraction, and in B8 firing during retraction (for protraction duration, $10.4 \pm 1.4 \mathrm{~s}$ vs $9.0 \pm 0.7 \mathrm{~s}, p=$ 0.53 ; for $\mathrm{B} 8$ frequency in protraction, $4.6 \pm$ $0.4 \mathrm{~Hz}$ vs $4.0 \pm 0.6 \mathrm{~s}, p=0.36$; for B8 frequency in retraction, $1.2 \pm 0.2 \mathrm{~Hz}$ vs $1.6 \pm$ $0.1 \mathrm{~s}, p=0.22$ ).

We found that hyperpolarization of CBI-5/6 did not extend the duration of the protraction phase of CBI-2-elicited egestive programs (Figs. $5 A, 6 C)\left(F_{(2,8)}=0.49\right.$, $p=0.63)$. This stands in sharp contrast to the protraction-extending actions of CBI5/6 hyperpolarization in EN-elicited egestive programs.

We also analyzed the timing of antidromic spikes recorded in CBI-5/6 soma. We found that in CBI-2-elicited egestive programs, in which CBI-5/6 were not hyperpolarized, the antidromic spikes appeared after the end of I2N activity (Fig. 5B1). The mean latency of the antidromic spikes in CBI-5/6 in CBI-2elicited egestive programs was positive $(67.8 \pm 23.4 \mathrm{~ms})$ (Fig. $6 E)$. This was consistent with the idea that CBI-5/6 may not act as a protraction terminator in these programs

The latencies of CBI-5/6 firing in the three types of programs that we studied (Figs. 3-5), i.e., CBI-2-elicited ingestive programs, CBI-2-elicited egestive programs, and EN-elicited egestive programs, differed significantly (Fig. $6 E)\left(F_{(2,20)}=15.31\right.$, $p<0.0001)$. The mean latency in EN-elicited egestive programs was significantly different from the mean latencies in CBI-2elicited ingestive programs $(p<0.001)$ and CBI-2-elicited egestive programs $(p<0.01)$. However, the difference between the mean latencies of CBI-2-elicited ingestive programs and CBI-2elicited egestive programs was not significant $(p=0.29)$. These results indicate that the functional importance of CBI-5/6 as a protraction terminator depends on the input that drives a specific program, but not on the type of the program that the $\mathrm{CPG}$ generates. 

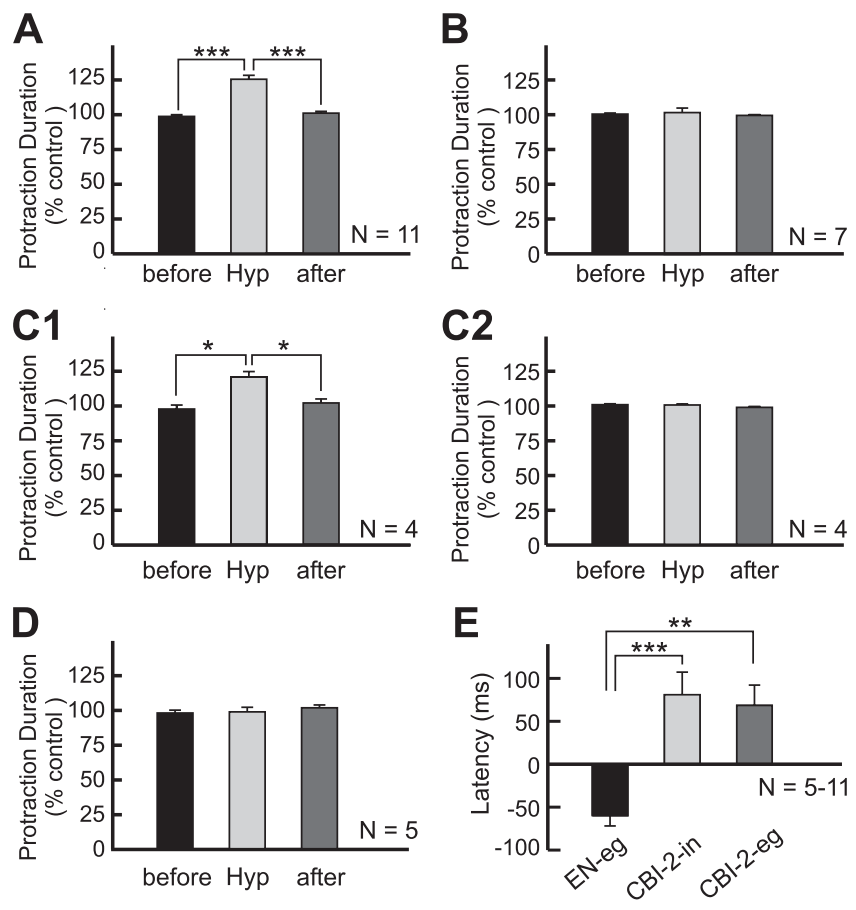

Figure 6. Grouped data of the effects of $\mathrm{CBI}-5 / 6$ hyperpolarization. $A$, The normalized protraction duration, before, during (Hyp), and after (BI-5/6 hyperpolarization in EN-elicited egestive programs (see Fig. 3). $\boldsymbol{B}$, The normalized protraction duration, before, during, and after CBI-5/6 hyperpolarization in CBI-2-elicited ingestive programs (see Fig. 4). C, The lengthening effect of $\mathrm{CBI}-5 / 6$ hyperpolarization is not caused by variability of different preparations we used. In four preparations, we examined the effects of $\mathrm{CBI}-5 / 6$ hyperpolarization both in EN-elicited egestive programs ( $\mathbf{C} \mathbf{1})$ and in $\mathrm{CBI}-2$-elicited ingestive programs $(\mathbf{C})$. Graphs in $\mathbf{C}$ and $\mathbf{C 2}$ represent a subset of data in $\boldsymbol{A}$ and $\boldsymbol{B}$, respectively. $\boldsymbol{D}$, The normalized protraction duration before, during, and after $\mathrm{CBI}-5 / 6$ hyperpolarization in CBI-2-elicited egestive programs (see Fig. 5). $\boldsymbol{E}$, The mean latencies of $\mathrm{CBI}-5 / 6$ firing relative to the termination of $\mathrm{I} 2 \mathrm{~N}$ activity in $\mathrm{EN}$ elicited egestive programs (EN-eg), in (BI-2-elicited ingestive programs (CBI-2-in), and in CBI2-elicited egestive programs (CBI-2-eg). Latency of $\mathrm{CBI}-5 / 6$ firing was calculated as the time that elapsed from the last extracellular signal peak recorded in I2N to the first peak of the antidromic spike recorded in $\mathrm{CBI}-5 / 6$ soma. If the first antidromic spike preceded the last signal in $12 \mathrm{~N}$, we defined the latency of $\mathrm{CBI}-5 / 6$ firing as negative, and if the first antidromic spike followed the last signal in $12 \mathrm{~N}$, we defined the latency of $\mathrm{CBI}-5 / 6$ firing as positive. ${ }^{*} p<0.05$; ${ }^{* *} p<0.01 ;{ }^{* * *} p<0.001$ (Bonferroni's post-test). Error bars indicate SEM.

\section{B65 regulates the spike onset timing of CBI-5/6 and B64 in an opposite manner}

Because CBI-5/6 fired sufficiently early in the protraction phase to act as a protraction terminator in egestive programs elicited via EN stimulation, but not in ingestive or egestive programs elicited via $\mathrm{CBI}-2$ stimulation, we reasoned that CBI-5/6 was likely to be regulated by a CPG element that is preferentially active in ENelicited egestive programs. Previous work has shown that (1) the CPG interneuron B65 (Kabotyanski et al., 1998), an inputrepresenting interneuron, is preferentially active in EN-elicited egestive programs, but not in CBI-2-elicited motor programs (Due et al., 2004; Jing and Weiss, 2005; Proekt et al., 2007); (2) when B65 is hyperpolarized in EN-elicited egestive programs, the hyperpolarization extends the duration of the protraction phase (Due et al., 2004); and (3) stimulation of B65 early in the protraction phase of CBI-2-elicited ingestive programs shortens the duration of protraction phase (Jing and Weiss, 2005). In view of these findings, it appeared plausible that B65 could act in EN-elicited egestive programs by advancing the timing of CBI-5/6 firing so that it begins to fire before the end of the protraction phase.

We, therefore, investigated whether B65 makes excitatory connections with CBI-5/6 within the buccal ganglia (Fig. 7A). We intracellularly stimulated neuron B65 while recording from the axon of CBI-5/6 $(n=3)$ and found that stimulation of B65 elicited fast depolarizing responses in the axon of CBI-5/6 (Fig. 7A). These responses followed one-for-one $\mathrm{B} 65$ action potentials, suggesting that the connection may be monosynaptic.

We then characterized the contribution of B65 to the timing of spike initiation of CBI-5/6 in motor programs that were elicited under different conditions. First, in EN-elicited egestive programs, we examined the effects of $\mathrm{B} 65$ hyperpolarization on the onset of CBI-5/6 firing relative to the end of I2N activity. Given that B65 hyperpolarization extended the duration of the protraction phase in EN-elicited egestive programs (Due et al., 2004), we reasoned that $\mathrm{B} 65$ hyperpolarization might delay the onset of CBI-5/6 firing relative to the end of the protraction phase. The representative result of such a hyperpolarization experiment is illustrated in Figure 7, $B$ and $C$. Figure $7 B$ shows the full motor programs and illustrates the effects of B65 hyperpolarization on the duration of protraction, and $C$ shows expanded records at the time of transition from the protraction to the retraction phase, and illustrates the timing of CBI-5/6 firing relative to the end of protraction. These egestive motor programs were elicited by EN stimulation, and B65 was recruited into these programs at high frequency (average spiking frequency, $\sim 9 \mathrm{~Hz}$ ). This was consistent with previous published data (Due et al., 2004; Proekt et al., 2007).

Similar to results reported previously, the duration of the protraction phase in motor programs in which B65 was hyperpolarized (Fig. 7B2) was longer than the duration of this phase in programs in which the membrane potential of $\mathrm{B} 65$ was not manipulated (Fig. 7B1,B3). The effects of B65 hyperpolarization on the protraction duration in EN-elicited egestive programs were statistically significant (Fig. $7 D)\left(F_{(2,6)}=10.70, p<0.01\right)$. More importantly, from the point of view of the present study, we found that B65 hyperpolarization indeed delayed the onset of CBI-5/6 firing relative to the end of $\mathrm{I} 2 \mathrm{~N}$ activity (Fig. $7 C$ ). The mean latencies before, during, and after B65 hyperpolarization were significantly different (Fig. $7 E)\left(F_{(2,6)}=7.70, p<0.05\right)$. Multiple-comparison tests showed that the mean CBI-5/6 latency during B65 hyperpolarization was significantly different from the before group $(p<0.05)$ and the after group $(p<0.05)$, whereas the difference between the before and the after groups was not significant $(p=0.88)$. Notably, the mean latencies between the onset of CBI-5/6 firing and the end of I2N activity were negative when B65 was not manipulated. In contrast, when neuron B65 was hyperpolarized, the mean latency became positive, thus apparently rendering CBI-5/6 incapable of acting as a protraction terminator.

If the protraction-terminating actions of CBI-5/6 indeed depend on the ability of B65 to excite CBI-5/6 axon, in principle, stimulation of $\mathrm{B} 65$ during the protraction phase of $\mathrm{CBI}-2$-elicited ingestive programs should advance the onset of CBI-5/6 firing relative to the termination of $\mathrm{I} 2 \mathrm{~N}$ activity. We tested this prediction in the experiments that are illustrated in Figure 8. We elicited ingestive programs via CBI-2 stimulation. In these programs, consistent with previous reports, B65 was only weakly active (average spiking frequency, $\sim 2 \mathrm{~Hz}$ ). B65 was fired at $14 \mathrm{~Hz}$ by injecting brief current pulses each of which elicited a single action potential (Fig. 8A2). Stimulation of B65 and CBI-2 was terminated after the protraction phase ended (i.e., when the activity in I $2 \mathrm{~N}$ ceased).

As reported previously (Jing and Weiss, 2005), stimulation of B65 shortened the duration of the protraction phase (Fig. 8A2), compared with the programs that were elicited before (Fig. $8 \mathrm{Al}$ ) 
A

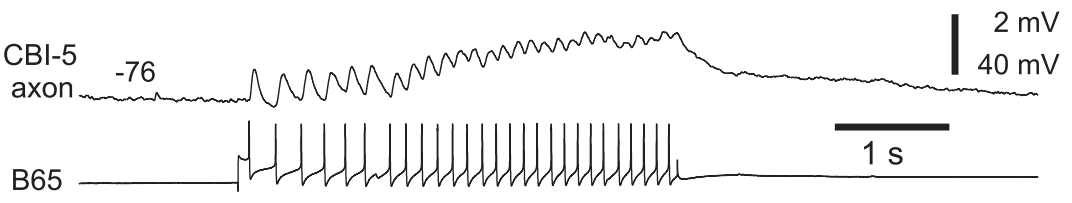

B1

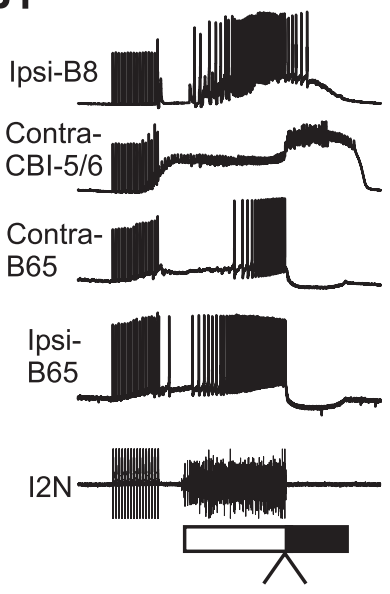

B2

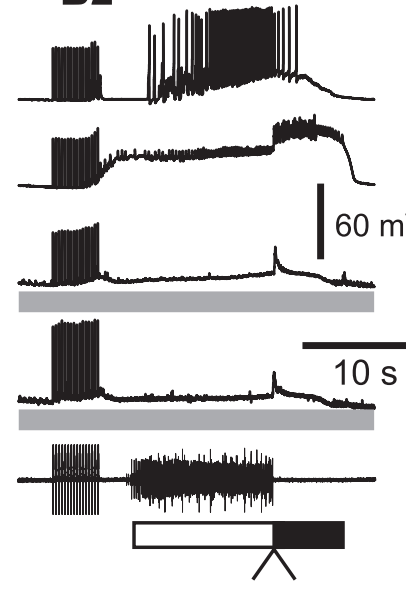

B3

C1

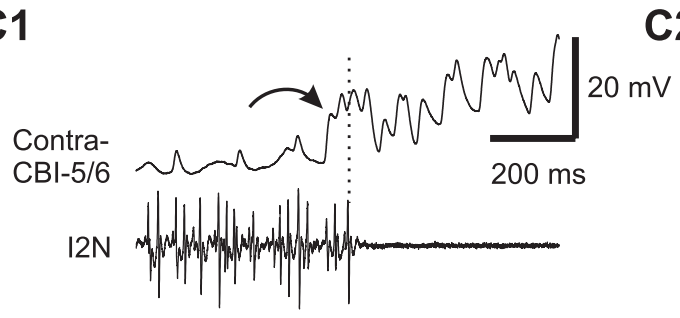

C2

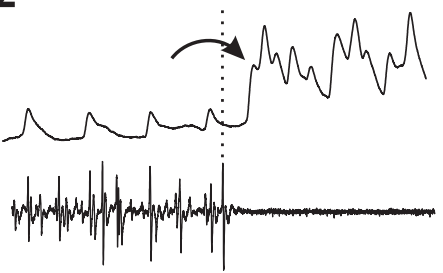

D

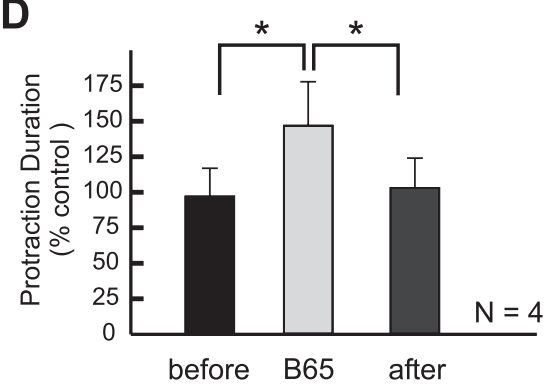

E

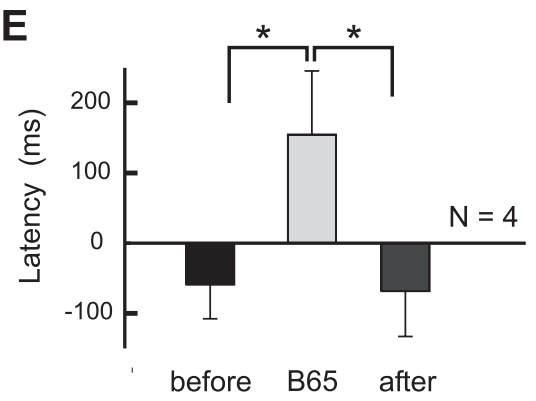

Figure 7. Effect of $\mathrm{B} 65$ hyperpolarization on the latency of $\mathrm{CBI}-5 / 6$ firing in EN-elicited egestive programs. $A$, Intracellular recordings from a CBI-5/6 axon and B65 in normal saline. B65 elicited one-for-one EPSPs in the CBI-5/6 axon. Note that B65elicited EPSPs in CBI-5/6 showed summation. B1, An EN-elicited egestive program in control condition. B2, Bilateral hyperpolarization of $\mathbf{B} 65$ extended the protraction duration. $\mathbf{B}$, The program after the hyperpolarization was released. $\mathbf{C}$, Expanded views of the transition between the protraction and retraction phases ( $B$ 1, $\boldsymbol{B} 2$, arrowheads) without $B 65$ hyperpolarization ( $\boldsymbol{C}$ 1 is from B1) and with B65 hyperpolarization ( 2 is from B2). Note that antidromic spikes in CBI-5/6 occurred before the end of I2N activity in the program in which $B 65 \mathrm{~s}$ were not hyperpolarized ( $\mathbf{C}$ ), whereas they began after the end of $\mathrm{I} 2 \mathrm{~N}$ activity when $\mathrm{B} 65 \mathrm{~s}$ were hyperpolarized (C2). D, Grouped data showing the normalized protraction duration before, during (B65), and after B65 hyperpolarization. $\boldsymbol{E}$, The mean latency of $(\mathrm{Bl}-5 / 6$ firing relative to the termination of $\mathrm{I} 2 \mathrm{~N}$ activity before, during (B65), and after B65 hyperpolarization. ${ }^{*} p<0.05$ (Bonferroni's post-test). Error bars indicate SEM.

and after (Fig. 8A3) the programs in which B65 was stimulated. The shortening actions of $\mathrm{B} 65$ stimulation were statistically significant (Fig. $8 C)\left(F_{(2,8)}=13.16, p<0.005\right)$. Importantly, B65 stimulation affected the onset of $\mathrm{CBI}-5 / 6$ firing relative to the termination of I2N activity (Fig. $8 B, D$ ). As illustrated in Figure 8 , $B 1$ and $B 2$, in which expanded records of the transition between protraction and retraction are shown, in motor programs that were elicited with and without a concurrent B65 stimulation, stimulation of B65 advanced the onset of CBI-5/6 firing relative to the end of protraction. Bonferroni's multiple-comparison test performed after the ANOVA (Fig. $8 D)\left(F_{(2,8)}=31.95, p<\right.$ 0.0005) showed that the mean CBI-5/6 latency during B65 stimulation was significantly different from the mean latencies of the before group $(p<0.001)$ and the after group ( $p<0.001)$, whereas the difference between the before and after groups was not significant $(p=0.76)$. Importantly, in these programs, when $\mathrm{B} 65$ was not stimulated, the latency of CBI-5/6 firing was positive, but became negative in programs in which $\mathrm{B} 65$ was stimulated (Fig. $8 D$ ), thus making CBI-5/6 a likely protraction terminator when $\mathrm{B} 65$ was stimulated.

These results, together with the previous findings (Sasaki et al., 2007) that direct stimulation of CBI-5/6 terminates prematurely the protraction phase and thereby shortens its duration, suggest that the level of activity of $\mathrm{B} 65$ may control the spike onset timing of CBI-5/6 and that through this mechanism, B65 may control whether CBI-5/6 acts as a protraction terminator.

Previous work showed that in addition to CBI-5/6, the feeding CPG also contains another protraction terminator, interneuron B64 (Hurwitz and Susswein, 1996), that, similar to CBI-5/6, is active in CBI-2elicited ingestive and, EN-elicited egestive programs (Wu et al., 2007). B64 was reported to receive fast EPSPs from B65 (Kabotyanski et al., 1998). Such a connection presumably should advance the firing of B64. Yet, this does not seem to happen, as in EN-elicited programs in which B65 fires at high frequency, B64 does not mediate protraction termination ( $\mathrm{Wu}$ et al., 2007). However, B64 does control protraction termination in CBI-2-elicited ingestive programs in which $\mathrm{B} 65$ does not fire at high frequency (Proekt et al., 2007; Wu et al., 2007).

To clarify this apparent contradiction between the nature of the B65-to-B64 connections and the functioning of B64 as a protraction terminator in circumstances in which B65 is active, we re-examined the characteristics of the synaptic connections between $\mathrm{B} 65$ and $\mathrm{B} 64$. Our reanalysis of the B65-to-B64 connections revealed that the connection, in addition to the previously reported fast excitatory component (Kabotyanski et al., 1998), also had an inhibitory component, which became more apparent when B65 was stimulated with a train (Fig. 9A). Specifically, during a train stimulation of B65, the inhibitory component summated. In contrast, the fast excitatory PSP decremented as reported previously (Kabotyanski et al., 1998) (Fig. 9A) $(n=5)$. To examine the effects of B65 on B64 excitability and, more specifically, on the timing of the onset of B64 firing, we stimulated B65 for $5 \mathrm{~s}$ until the current injection into B64 (2.5 s), which was applied every $30 \mathrm{~s}$, was begun. This 
stimulation paradigm is designed to simulate the order by which these neurons are recruited into motor programs, i.e., B65 and B64 fire during protraction and retraction, respectively. The amplitude of current pulses injected into B64 was adjusted to elicit 10-14 spikes during current injection. This experiment was conducted in $\mathrm{Hi}$-Di to reduce confounding contributions of polysynaptic pathways.

In Figure 9B, 12 spikes in B64 without B65 stimulation were reduced to eight when $\mathrm{B} 65$ was stimulated $(\sim 33 \%$ reduction). The average numbers of B64 spikes before, during, and after B65 manipulation were significantly different (Fig. 9C) $\left(F_{(2,8)}\right.$ $=19.70, p<0.001)$. The mean number of B64 spikes during B65 manipulation was significantly different from the before group $(p<0.01)$ and the after group $(p<$ 0.01 ), whereas the difference between the before and the after groups was not significant $(p=0.65)$. More importantly, B65 stimulation increased the latency between the onset of current injection and the appearance of the first action potential in B64. In Figure 9B, the latency of $106.6 \mathrm{~ms}$ in controls became 632.0 ms after B65 stimulation ( $\sim 493 \%$ increase). The overall difference between the groups, before, during, and after B65 manipulation was statistically significant (Fig. 9D) $\left(F_{(2,8)}=6.69\right.$, $p<0.05)$. B65 stimulation significantly delayed the onset of B64 firing compared with the before $(p<0.05)$ and the after $(p<$ 0.05) B65 manipulation, whereas the difference between the before and after conditions was not significant ( $p=0.93$ ). This suggests that in motor programs, in which $\mathrm{B} 65$ is active, the onset of B64 firing may be delayed.

To investigate whether in motor programs, in which B65 is active, the onset of B64 firing is delayed, we examined the effect of B65 stimulation on the latency of $\mathrm{B} 64$ firing relative to the termination of I2N activity during CBI-2-elicited ingestive programs (Fig. 10). During this type of program, B65 is less active (Jing and Weiss, 2005; Proekt et al., 2007) (Figs. 8 A, 10A), and B64 begins to fire spikes before the termination of the protraction phase (Wu et al., 2007) (Fig. 10). If the inhibitory connection of B65 to B64 was functionally effective, then B65 stimulation should delay the onset of B64 firing. We tested this prediction in the experiments that are illustrated in Figure 10. As shown above (Fig. 8), B65 stimulation again significantly shortened the duration of the protraction phase (Fig. $10 A, C)\left(F_{(2,6)}=10.04, p<0.05\right)$. Importantly, as predicted, stimulation of B65 delayed the onset of B64 firing relative to the end of the protraction phase (Fig. $10 \mathrm{~B}$ ). The Bonferroni's multiple-comparison tests that followed the ANOVA (Fig. 10D) $\left(F_{(2,6)}=26.49, p<0.005\right)$ showed that the mean B64 latency during B65 stimulation was significantly different from the before $(p<0.01)$ and the after condition $(p<0.01)$, whereas the difference between the before and after conditions was not
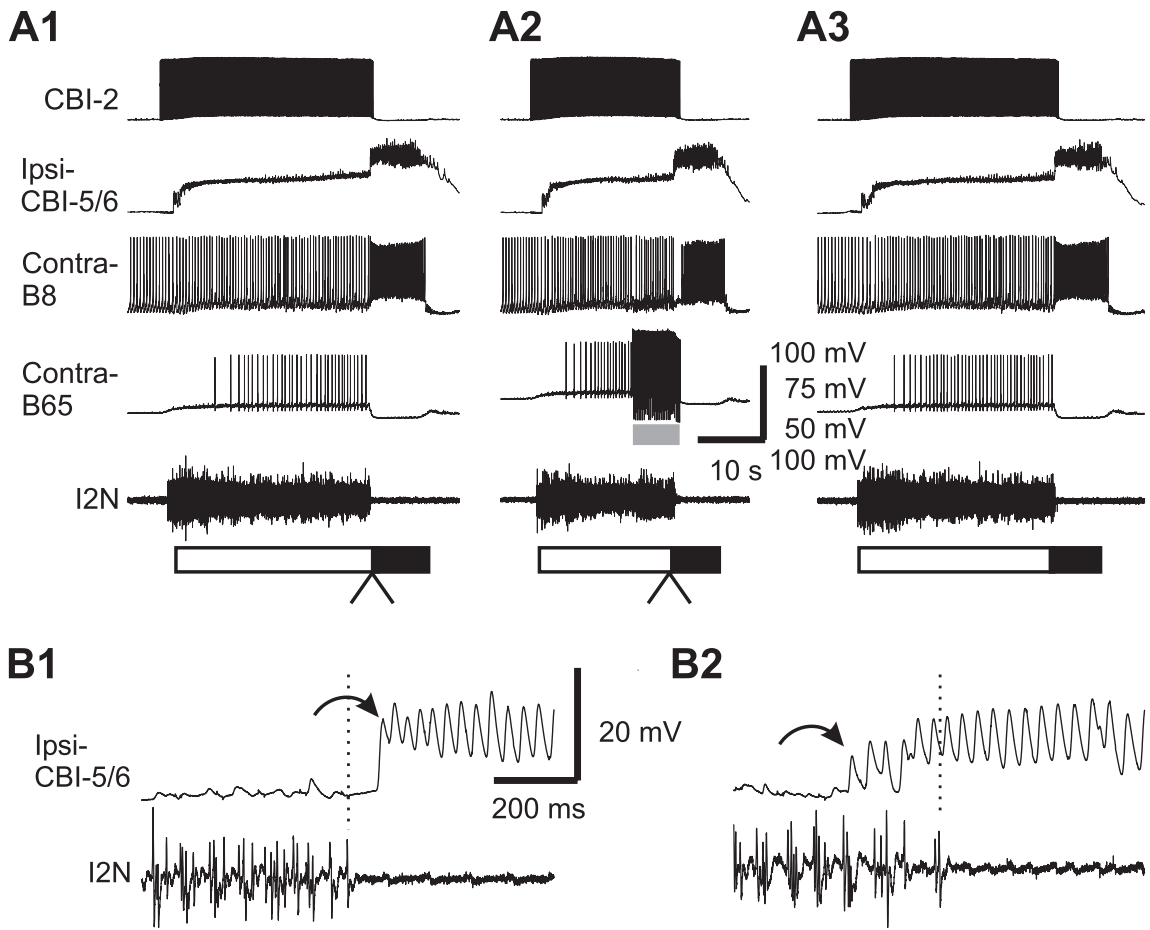

B2
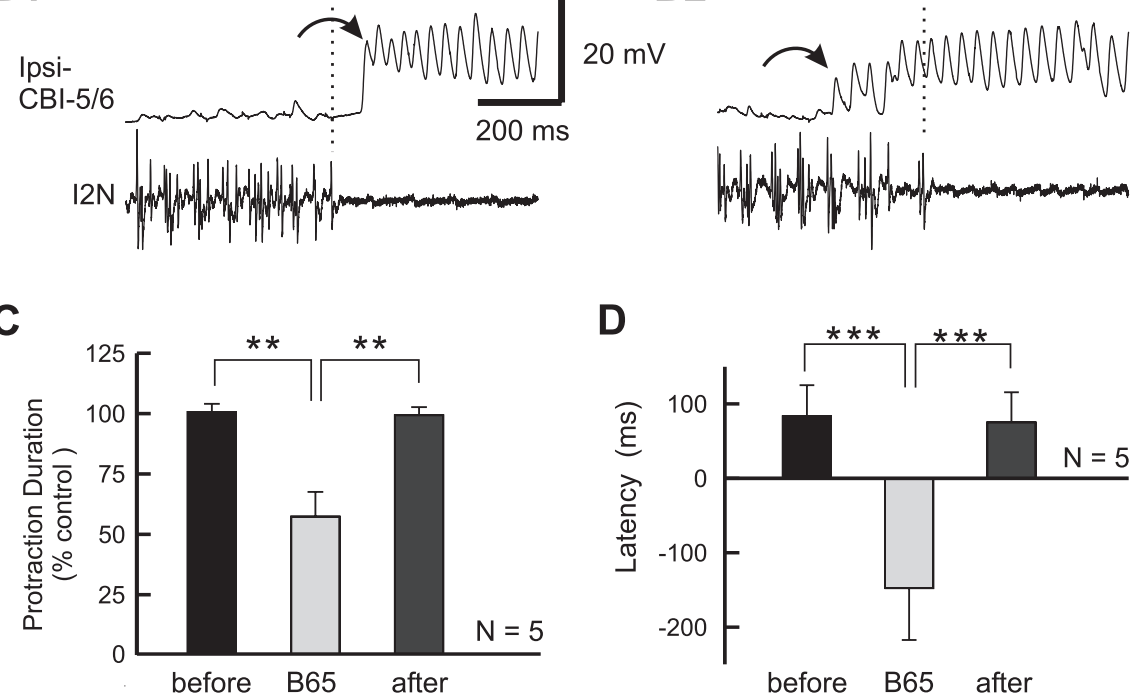

Figure 8. Effect of $\mathrm{B} 65$ stimulation on the latency of $\mathrm{CBI}-5 / 6$ firing in $\mathrm{CBI}-2$-elicited ingestive programs. A1, $\mathrm{A}$ CBI-2-elicited ingestive program in control condition. $\mathbf{A 2}$, Stimulation of $\mathrm{B} 65$ at $14 \mathrm{~Hz}$ during protraction terminated the phase before the (2) antidromic spikes in $\mathrm{CBI}-5 / 6$ began after the end of $2 \mathrm{~N}$ activity in the control (B1), whereas they began before the end a when $B 65$ was stimulated (B2).C, Grouped data showing the normalized protraction duration before, during (B65), and after $\mathrm{B} 65$ stimulation. $\boldsymbol{D}$, The mean latency of $\mathrm{CBI}-5 / 6$ firing relative to the termination of $\mathrm{I} 2 \mathrm{~N}$ activity before, during, and after B65 stimulation. ${ }^{* *} p<0.01 ;{ }^{* *} p<0.001$ (Bonferroni's post-test). Error bars indicate SEM.

significant $(p=0.80)$. In these programs, the latency of B64 firing was negative in controls, but became positive when $\mathrm{B} 65$ was stimulated (Fig. 10D). This stands in sharp contrast to the effects that stimulation of $\mathrm{B} 65$ exerts on the latency of CBI-5/6 firing (Fig. $8 D$ ). In principle, this could provide an additional means for allowing CBI-5/6 to assume the role of a protraction terminator when B65 is active, as is the case in EN-elicited egestive programs.

\section{Discussion}

Our results indicate that phase-switching functions in the Aplysia feeding network are mediated by a population of neurons that include B64 and CBI-5/6. Furthermore, we showed that a CPG interneuron $\mathrm{B} 65$ regulates the spike timing of $\mathrm{B} 64$ and CBI-5/6 and thereby the selection of which of these neurons acts as a protraction terminator.

Several lines of evidence suggest that CBI-5/6 implements protraction termination in a B64-independent manner. First, 

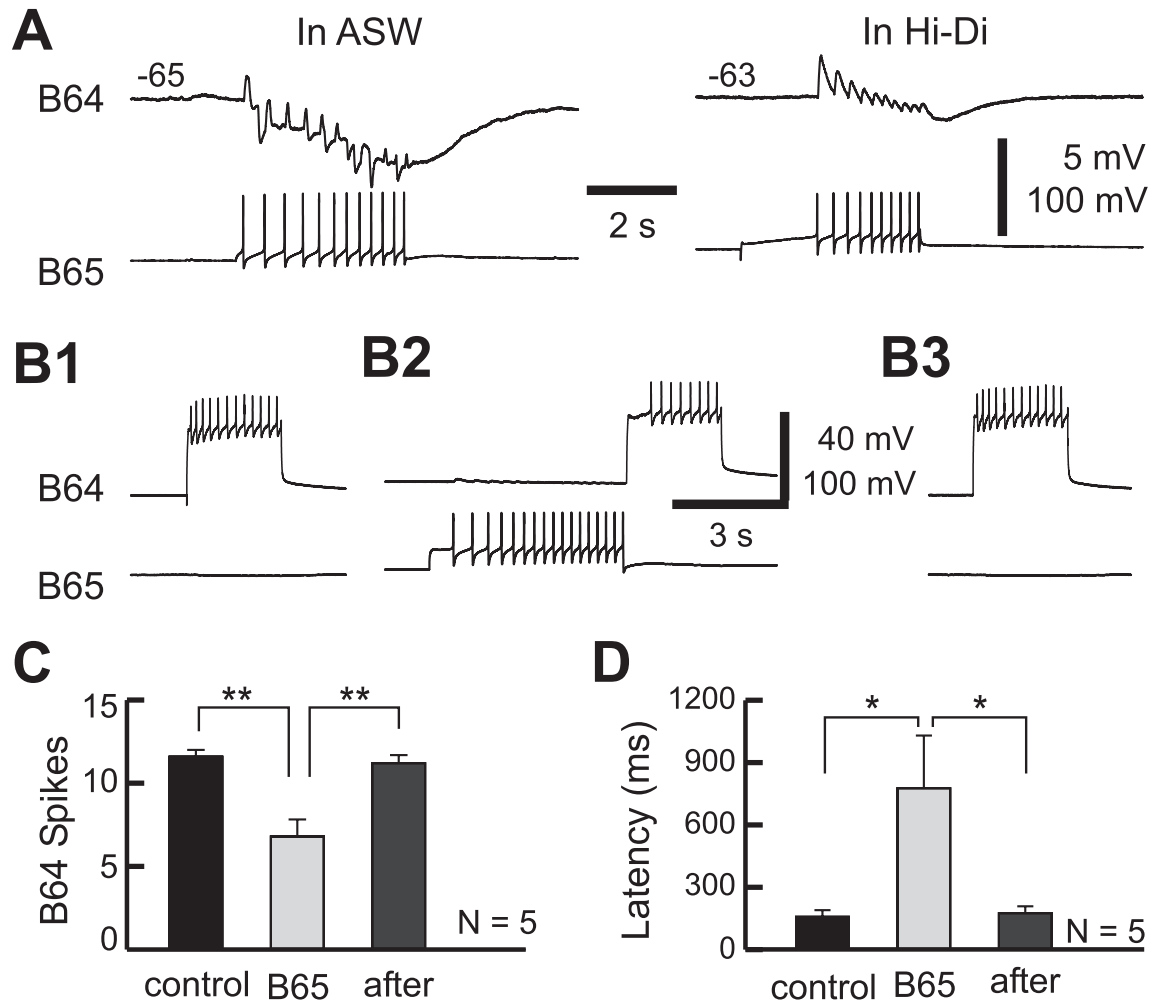

Figure 9. B65 decreases the excitability of interneuron B64.A, B65 elicited not only the one-for-one fast EPSPs, but also a slow inhibitory response in the contralateral B64 in normal saline (left). Both PSP components persisted in Hi-Di (right). B, Stimulation of $B 65$ reduced the number of spikes in B64 elicited by depolarizing current pulses ( $2.5 \mathrm{~s}$ duration) every $60 \mathrm{~s}$. Without B65 stimulation $(\boldsymbol{B} 1, \boldsymbol{B} 3)$, the current pulse in $B 64$ induced 12 spikes in $B 64$. When $B 65$ was stimulated for $5 \mathrm{~s}$ before the current injection into $B 64$, the number of $B 64$ spikes was reduced to eight (B2). Note that compared with the control, prestimulation of $B 65$ also delayed the onset of $B 64$ firing relative to the onset of current injection into it. $C$, Grouped data showing the mean number of B64 spikes elicited by a constant current injection before, during (B65), and after B65 stimulation. $\boldsymbol{D}$, The mean latencies between the onset of current injection into $B 64$ and the appearance of the first action potential in $B 64$ before, during (B65), and after B65 stimulation. ${ }^{*} p<0.05 ;{ }^{* *} p<0.01$ (Bonferroni's post-test). Error bars indicate SEM.

similar to B64, CBI-5/6 is recruited into both ingestive and egestive programs and displays a similar firing pattern and frequency in both programs (Sasaki et al., 2007). Second, the apparently glutamatergic synaptic connections of both B64 and CBI-5/6 (Due et al., 2005; Sasaki et al., 2007) act to suppress the protraction phase and promote the retraction phase of motor programs. Third, CBI-5/6 stimulation early in the protraction phase terminates this phase (Sasaki et al., 2007). However, the soma of the compartmentalized CBI-5/6 is unlikely to generate full-size action potentials. Thus, it was suggested that the action potentials originate in the axon and then propagate toward the soma (Perrins and Weiss, 1998; Sasaki et al., 2007). Using simultaneous somatic and axonal recording, we demonstrated that this is the case during motor programs, thus supporting the idea that the buccal region of CBI-5/6 acts as an element of the CPG. The ability of the remote axon and its terminal to generate action potentials and to influence motor patterns was observed in other systems (Bartos and Nusbaum, 1997; Bucher et al., 2003).

\section{Spike timing and phase-switching function: input dependence and neural mechanism}

Previous work (Wu et al., 2007) indicated that B64 spike timing, which is critical to its phase-switching function, may be regulated by the state of the network. Specifically, our previous work showed that (1) B64 is a protraction terminator in CBI-2-elicited ingestive programs, and (2) B64 is not a protraction terminator when egestive programs are elicited via CBI-2 and EN. Because B64 is a protraction terminator in ingestive but not egestive programs, and this selectivity is not input dependent, we suggested that either the state of the network or the type of program determines whether B64 is a protraction terminator. Here, we show that CBI-5/6 is a protraction terminator when egestive programs are elicited via EN, but not via CBI-2. Therefore, whether CBI-5/6 functions as a protraction terminator is input dependent. The combined actions of CBI-5/6 and B64 can account for protraction termination in CBI-2-elicited ingestive programs and in EN-elicited egestive programs, but not for protraction termination in CBI-2-elicited egestive programs. Thus, although B64 and CBI-5/6 are in some way complementary to each other, an additional unidentified neuron(s) may mediate protraction termination in CBI-2-elicited egestive programs. Be that as it may, the finding that the spike timing and the protraction-terminating function of CBI-5/6 are input dependent led to the question of how B64 or CBI-5/6 is differentially selected to act as a protraction terminator when different inputs activate the network.

A CPG interneuron, B65, is active in EN-elicited egestive programs but not in CBI-2-elicited egestive or ingestive programs, and B65 is thus considered to be an input-representing neuron (Proekt et al., 2007). Notably, B65 is active when CBI-5/6 is a protraction terminator, but is not active when CBI-5/6 is not a terminator. Furthermore, in EN-elicited egestive programs, when we suppressed B65 activity, the onset of CBI-5/6 firing relative to protraction termination was delayed and actually began after the end of protraction. Additionally, B65 simultaneously modulated CBI-5/6 and B64 spike timing in opposite directions. Thus, B65 stimulation in CBI-2-elicited ingestive programs, in which B64 normally acts a protraction terminator (Wu et al., 2007), advanced CBI-5/6 firing so that it began to fire before protraction termination, whereas B64 firing was delayed and it began to fire after protraction termination. In effect, by stimulating B65, we substituted the protraction-terminating role of $\mathrm{B} 64$ with $\mathrm{CBI}-5 / 6$. The pattern of synaptic connections from B65 to CBI-5/6 and to B64 is entirely consistent with advancing and delaying actions that B65 exerts respectively on CBI-5/6 and B64. In CBI-5/6, B65 elicits fast summating EPSPs that bring CBI-5/6 closer to threshold. In B64, B65 elicits complex PSPs that function to delay the onset of B64 firing. In Figure 11, we illustrate the current understanding of the mechanisms of the selection of B64 versus CBI-5/6 as a protraction terminator.

The fact that, in programs elicited by CBI-2, B64 may or may not act as a protraction terminator suggests that the protractionterminating function of B64 is not input dependent. At the same time, B64 is not a protraction terminator when B65 is active. In turn B65 is activated by a specific input, EN. This may suggest that the protraction-terminating function of B64 is input dependent. This apparent paradox disappears, however, if one accepts 
the possibility that B65 is not the only neuron that can affect the onset of B64 firing. Indeed, our data suggest that in CBI-2elicted egestive programs, an additional neuron that acts as a protraction terminator is recruited to fire before B64 (see above).

A number of studies emphasized the importance of higher-order neurons in differentially configuring the activity of CPG elements (Blitz et al., 1999; Combes et al., 1999; Kupfermann and Weiss, 2001; Zelenin et al., 2001; Jing et al., 2004; Kristan et al., 2005). The use of higher-order neurons may provide a simple means of implementing the input-dependent selection of phase-transition neurons. Indeed, CBI-2 enhances B64 excitability (Koh and Weiss, 2007). In contrast, we show that the input dependence of CBI-5/6 functioning as a phase switch may be primarily mediated by an input-representing CPG neuron, B65 (Fig. 11). B65 actions suggest that inputrepresenting neurons that are themselves elements of the CPG may play a role similar to that attributed to higher-order neurons. In addition, because B65 activity may be further regulated by other inputs, the mechanisms described in here may support additional forms of network and behavior flexibility (Jing and Weiss, 2005; Lum et al., 2005; Proekt et al., 2005; Zhurov et al., 2005; Jing et al., 2007).

\section{Generation of multiple outputs}

The Aplysia feeding network generates several motor outputs, and it shares a number of organizational features with other multifunctional CPGs. One such feature is the existence of two classes of CPG elements, the shared and nonshared CPG elements. The shared elements were defined as those active in different classes of motor programs, whereas the nonshared ones are preferentially active in a subset of motor programs (Heitler, 1985; Getting, 1989; Soffe, 1993; Berkowitz and Stein, 1994; Marder and Calabrese, 1996; Shaw and Kristan, 1997; Lieske et al., 2000; Jing and Weiss, 2001; Jing et al., 2004; Briggman and Kristan, 2006; Berkowitz, 2007; Proekt et al., 2007). Although presumably shared neurons fulfill the same function in differing programs in which they are active, previous data indicate that shared neurons may implement one of their specific functions only in a subset of programs and not fulfill this function in other programs (Ayali and Harris-Warrick, 1999; Thirumalai et al., 2006; Saideman et al., 2007; Wu et al., 2007).

In the Aplysia feeding CPG, motoneurons that implement the protraction/retraction movements are activated in a fixed sequence, in which protraction always precedes retraction, and are thus considered to be phase fixed. However, motoneurons that implement the opening/closing movements switch their phasing so that in ingestive programs the radula closers are preferentially active in retraction, whereas in egestive programs they are predominantly active in protraction (Morton and Chiel, 1993a,b).
A2

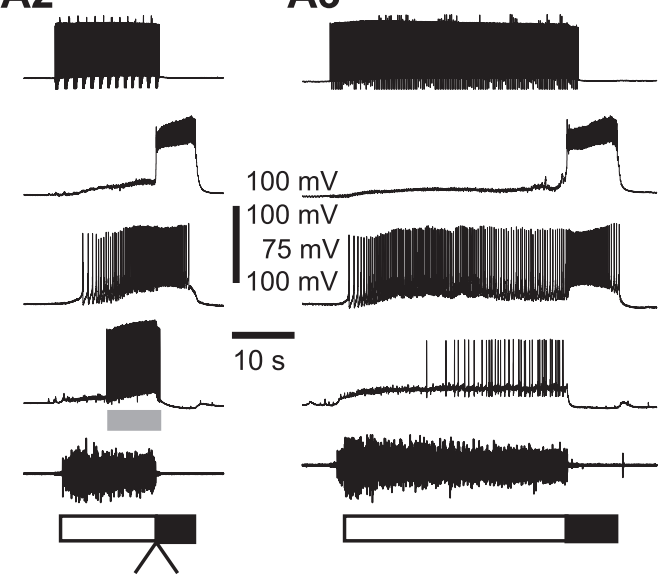

B2
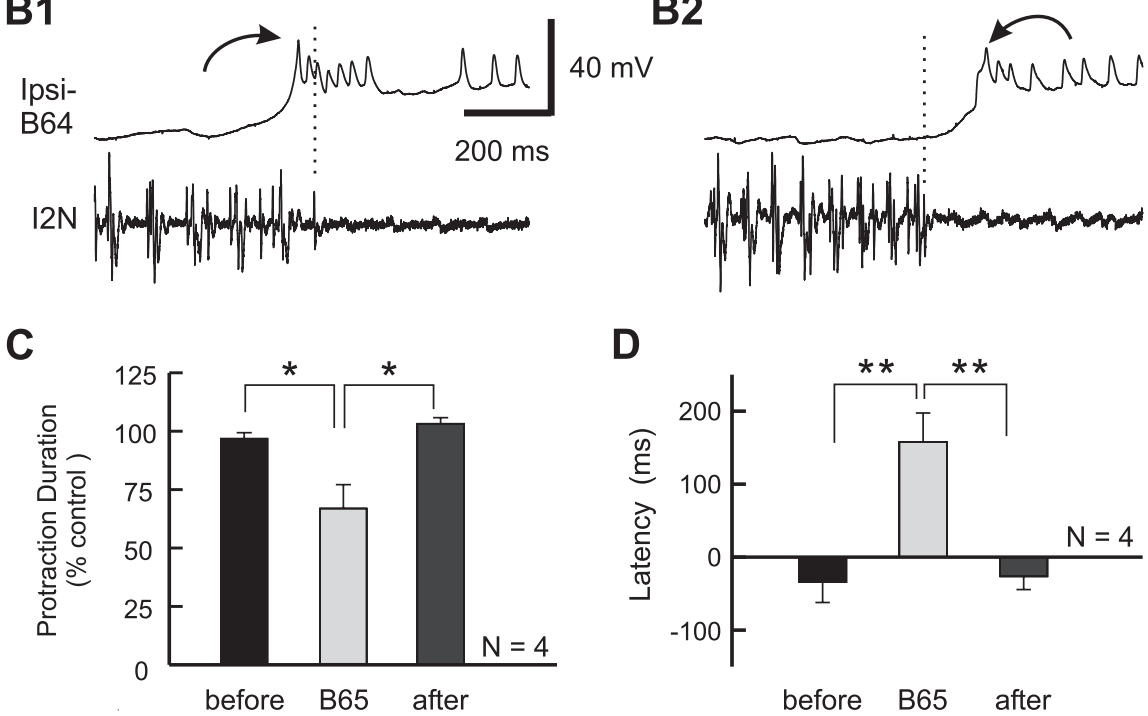

Figure 10. Effect of $\mathrm{B} 65$ stimulation on the latency of $\mathrm{B} 64$ firing in $\mathrm{CBI}-2$-elicited ingestive programs. A1, A CBI-2-elicited 政 stimulation. $\boldsymbol{D}$, The mean latency of $B 64$ firing relative to the termination of I2N activity before, during (B65), and after B65 stimulation. ${ }^{*} p<0.05 ;{ }^{* *} p<0.01$ (Bonferroni's post-test). Error bars indicate SEM.

These neurons are thus considered to be phase shifting. Consistent with this organizational feature, radula closing motoneurons are controlled by nonshared CPG interneurons (Jing and Weiss, 2001; Jing et al., 2003, 2004; Proekt et al., 2007). In contrast, the CPG interneurons which belong to the class of shared interneurons control the phase-fixed protraction/retraction motoneurons (Jing et al., 2004; Sasaki et al., 2007; Wu et al., 2007). In principle, different combinations of activity of shared and nonshared CPG elements could give rise to distinct but related motor outputs. In such a view, shared interneurons would produce a similar pattern of activity of the protraction/retraction motoneurons in ingestive and egestive programs, whereas the nonshared interneurons would produce differing firing patterns of opening/ closing motoneurons in different programs. However, this view may be an oversimplification as different shared neurons, CBI$5 / 6$ and B64, act as protraction terminators in different motor programs. Specifically, the functional change results not from a radical change in activity levels or patterns, as most commonly 
A EN-elicited egestive programs
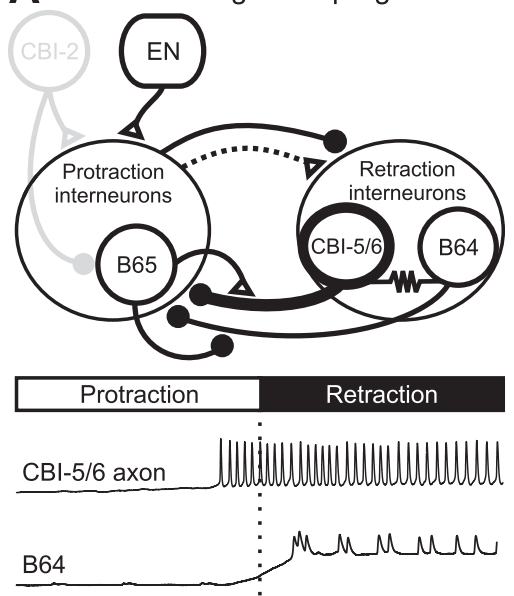

A : Excitatory connection
B CBI-2-elicited ingestive programs

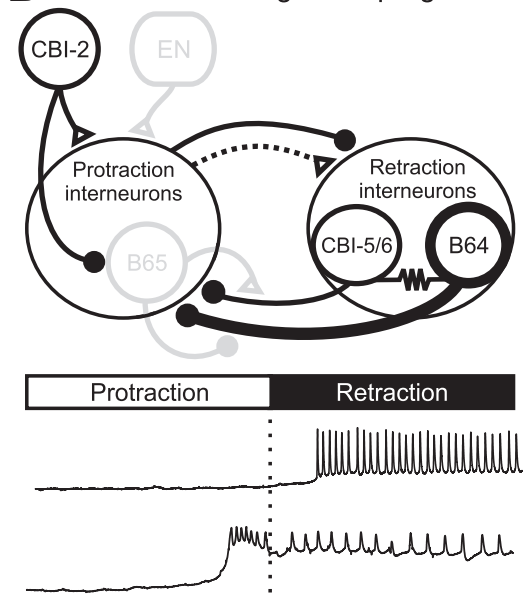

Inhibitory connection $\quad-$ - Electrical connection

Figure 11. A schematic diagram illustrating synaptic mechanisms that an input-representing interneuron B65 uses to regulate the spike timing and the phase-switching function of $\mathrm{CBI}-5 / 6$ and $\mathrm{B} 64$. Both $\mathrm{CBI}-5 / 6$ and $\mathrm{B} 64$ receive fast excitatory inputs from B65, but $B 64$ also receives prominent slow inhibitory inputs. Here, only the inhibitory connection from B65 to B64 is depicted because the mixed excitatory and inhibitory actions of B65 functionally decrease B64 excitability. In addition, both (BI-5/6 (Sasaki et al., 2007) and B64 (Hurwitz and Susswein, 1996; Kabotyanski et al., 1998) make inhibitory synaptic connections with protraction neurons including B65. A, In EN-elicited egestive programs, B65 is activated at high frequency (Proekt et al., 2007), thereby advancing the timing of (BI-5/6 firing, but delaying the timing of B64 firing (lower electrophysiological recordings). Thus, in this type of program, $\mathrm{CB}-5 / 6$, but not $\mathrm{B} 64$ functions as a protraction terminator by inhibiting protraction neurons (Sasaki et al., 2007; present study). B, In CBI-2-elicited ingestive programs, B65 is inhibited by (BI-2 (Jing and Weiss, 2005). In the absence of excitation of the $\mathrm{CBI}-5 / 6$ axon by $\mathrm{B} 65$, the timing of $\mathrm{CBI}-5 / 6$ firing may be delayed. In contrast, without $\mathrm{B} 65$ inhibition, B64 fires early, in part because of slow excitation from protraction interneurons, such as B63, and excitation from neuropeptides released by CBI-2 (Koh and Weiss, 2007). Thus, in this type of program, B64, but not CBI-5/6, functions as a protraction terminator by inhibiting protraction neurons.

observed (Getting and Dekin, 1985; Hooper and Moulins, 1989; Dickinson et al., 1990; Popescu and Frost, 2002; Jing and Gillette, 2003), but from a change in relative spike timing. It is worth noting, though, that B64 and CBI-5/6 may fulfill additional functions, e.g., excitation of retraction neurons, even in those programs in which these neurons do not act as protraction terminators.

In summary, we characterized one neural substrate that underlies regulation of spike timing and neuronal function. It is attractive to think that, because of their simplicity, the mechanisms that we have identified here may also be exploited by other networks.

\section{References}

Ayali A, Harris-Warrick RM (1999) Monoamine control of the pacemaker kernel and cycle frequency in the lobster pyloric network. J Neurosci 19:6712-6722.

Bartos M, Nusbaum MP (1997) Intercircuit control of motor pattern modulation by presynaptic inhibition. J Neurosci 17:2247-2256.

Berkowitz A (2007) Spinal interneurons that are selectively activated during fictive flexion reflex. J Neurosci 27:4634-4641.

Berkowitz A, Stein PS (1994) Activity of descending propriospinal axons in the turtle hindlimb enlargement during two forms of fictive scratching: phase analyses. J Neurosci 14:5105-5119.

Bi GQ, Poo MM (1998) Synaptic modifications in cultured hippocampal neurons: dependence on spike timing, synaptic strength, and postsynaptic cell type. J Neurosci 18:10464-10472.

Blitz DM, Christie AE, Coleman MJ, Norris BJ, Marder E, Nusbaum MP (1999) Different proctolin neurons elicit distinct motor patterns from a multifunctional neuronal network. J Neurosci 19:5449-5463.

Briggman KL, Kristan Jr WB (2006) Imaging dedicated and multifunctional neural circuits generating distinct behaviors. J Neurosci 26:10925-10933.

Bucher D, Thirumalai V, Marder E (2003) Axonal dopamine receptors ac- tivate peripheral spike initiation in a stomatogastric motor neuron. J Neurosci 23:6866-6875.

Cassenaer S, Laurent G (2007) Hebbian STDP in mushroom bodies facilitates the synchronous flow of olfactory information in locusts. Nature 448:709-713.

Church PJ, Lloyd PE (1994) Activity of multiple identified motor neurons recorded intracellularly during evoked feeding-like motor programs in Aplysia. J Neurophysiol 72:1794-1809.

Combes D, Meyrand P, Simmers J (1999) Dynamic restructuring of a rhythmic motor program by a single mechanoreceptor neuron in lobster. J Neurosci 19:3620-3628.

Dickinson PS, Mecsas C, Marder E (1990) Neuropeptide fusion of two motor-pattern generator circuits. Nature 344:155-158.

Due MR, Jing J, Weiss KR (2004) Dopaminergic contributions to modulatory functions of a dual-transmitter interneuron in Aplysia. Neurosci Lett 358:53-57.

Due MR, Alexeeva V, Jing J, Weiss KR, Vilim F (2005) Involvement of glutamate in specification of protraction phase duration in feeding motor programs of Aplysia. Soc Neurosci Abstr 31:177.10.

Getting PA (1989) Reconstruction of small neural networks. In: Methods in neuronal modeling (Koch C, Segev I, eds), pp 171-194. Cambridge, MA: MIT.

Getting PA, Dekin MS (1985) Mechanisms of pattern generation underlying swimming in Tritonia. IV. Gating of central pattern generator. J Neurophysiol 53:466-480.

Heitler WJ (1985) Motor programme switching in the crayfish swimmeret system. J Exp Biol 114:521-549.

Hooper SL, Moulins M (1989) Switching of a neuron from one network to another by sensory-induced changes in membrane properties. Science 244:1587-1589.

Hurwitz I, Susswein AJ (1996) B64, a newly identified central pattern generator element producing a phase switch from protraction to retraction in buccal motor programs of Aplysia californica. J Neurophysiol 75:1327-1344.

Hurwitz I, Goldstein RS, Susswein AJ (1994) Compartmentalization of pattern-initiation and motor functions in the B31 and B32 neurons of the buccal ganglia of Aplysia californica. J Neurophysiol 71:1514-1527.

Hurwitz I, Neustadter D, Morton DW, Chiel HJ, Susswein AJ (1996) Activity patterns of the B31/B32 pattern initiators innervating the I2 muscle of the buccal mass during normal feeding movements in Aplysia californica. J Neurophysiol 75:1309-1326.

Jing J, Gillette R (2003) Directional avoidance turns encoded by single interneurons and sustained by multifunctional serotonergic cells. J Neurosci 23:3039-3051.

Jing J, Weiss KR (2001) Neural mechanisms of motor program switching in Aplysia. J Neurosci 21:7349-7362.

Jing J, Weiss KR (2005) Generation of variants of a motor act in a modular and hierarchical motor network. Curr Biol 15:1712-1721.

Jing J, Vilim FS, Wu JS, Park JH, Weiss KR (2003) Concerted GABAergic actions of Aplysia feeding interneurons in motor program specification. J Neurosci 23:5283-5294.

Jing J, Cropper EC, Hurwitz I, Weiss KR (2004) The construction of movement with behavior-specific and behavior-independent modules. J Neurosci 24:6315-6325.

Jing J, Vilim FS, Horn CC, Alexeeva V, Hatcher NG, Sasaki K, Yashina I, Kupfermann I, Sweedler JV, Weiss KR (2007) From hunger to satiety: reconfiguration of a feeding network by Aplysia neuropeptide Y. J Neurosci 27:3490-3502.

Kabotyanski EA, Baxter DA, Byrne JH (1998) Identification and characterization of catecholaminergic neuron B65, which initiates and modifies 
patterned activity in the buccal ganglia of Aplysia. J Neurophysiol 79:605-621.

Koh HY, Weiss KR (2007) Activity-dependent peptidergic modulation of the plateau-generating neuron B64 in the feeding network of Aplysia. J Neurophysiol 97:1862-1867.

Kristan Jr WB, Calabrese RL, Friesen WO (2005) Neuronal control of leech behavior. Prog Neurobiol 76:279-327.

Kupfermann I, Weiss KR (2001) Motor program selection in simple model systems. Curr Opin Neurobiol 11:673-677.

Lieske SP, Thoby-Brisson M, Telgkamp P, Ramirez JM (2000) Reconfiguration of the neural network controlling multiple breathing patterns: eupnea, sighs and gasps. Nat Neurosci 3:600-607.

Lum CS, Zhurov Y, Cropper EC, Weiss KR, Brezina V (2005) Variability of swallowing performance in intact, freely feeding Aplysia. J Neurophysiol 94:2427-2446.

Marder E, Calabrese RL (1996) Principles of rhythmic motor pattern generation. Physiol Rev 76:687-717.

Morgan PT, Jing J, Vilim FS, Weiss KR (2002) Interneuronal and peptidergic control of motor pattern switching in Aplysia. J Neurophysiol $87: 49-61$

Morton DW, Chiel HJ (1993a) The timing of activity in motor neurons that produce radula movements distinguishes ingestion from rejection in Aplysia. J Comp Physiol 173:519-536.

Morton DW, Chiel HJ (1993b) In vivo buccal nerve activity that distinguishes ingestion from rejection can be used to predict behavioral transitions in Aplysia. J Comp Physiol 172:17-32.

Murphy GJ, Rieke F (2006) Network variability limits stimulus-evoked spike timing precision in retinal ganglion cells. Neuron 52:511-524.

Nargeot R, Baxter DA, Byrne JH (1997) Contingent-dependent enhancement of rhythmic motor patterns: an in vitro analog of operant conditioning. J Neurosci 17:8093-8105.

Perrins R, Weiss KR (1998) Compartmentalization of information processing in an Aplysia feeding circuit interneuron through membrane properties and synaptic interactions. J Neurosci 18:3977-3989.

Popescu IR, Frost WN (2002) Highly dissimilar behaviors mediated by a multifunctional network in the marine mollusk Tritonia diomedea. J Neurosci 22:1985-1993.

Proekt A, Brezina V, Weiss KR (2004) Dynamical basis of intentions and expectations in a simple neuronal network. Proc Natl Acad Sci USA 101:9447-9452.

Proekt A, Jing J, Weiss KR (2007) Multiple contributions of an inputrepresenting neuron to the dynamics of the Aplysia feeding network. J Neurophysiol 97:3046-3056.
Proekt A, Vilim FS, Alexeeva V, Brezina V, Friedman A, Jing J, Li L, Zhurov Y, Sweedler JV, Weiss KR (2005) Identification of a new neuropeptide precursor reveals a novel source of extrinsic modulation in the feeding system of Aplysia. J Neurosci 25:9637-9648.

Robbe D, Montgomery SM, Thome A, Rueda-Orozco PE, McNaughton BL, Buzsaki G (2006) Cannabinoids reveal importance of spike timing coordination in hippocampal function. Nat Neurosci 9:1526-1533.

Roberts PD, Bell CC (2002) Active control of spike-timing dependent synaptic plasticity in an electrosensory system. J Physiol (Paris) 96:445-449.

Rosen SC, Teyke T, Miller MW, Weiss KR, Kupfermann I (1991) Identification and characterization of cerebral-to-buccal interneurons implicated in the control of motor programs associated with feeding in Aplysia. J Neurosci 11:3630-3655.

Saideman SR, Blitz DM, Nusbaum MP (2007) Convergent motor patterns from divergent circuits. J Neurosci 27:6664-6674.

Sakurai A, Darghouth NR, Butera RJ, Katz PS (2006) Serotonergic enhancement of a 4-AP-sensitive current mediates the synaptic depression phase of spike timing-dependent neuromodulation. J Neurosci 26:2010-2021.

Sanchez JA, Kirk MD (2000) Short-term synaptic enhancement modulates ingestion motor programs of Aplysia. J Neurosci 20:RC 85:1-7.

Sasaki K, Due MR, Jing J, Weiss KR (2007) The feeding CPG in Aplysia directly controls two distinct outputs of a compartmentalized interneuron that functions as a CPG element. J Neurophysiol 98:3796-3801.

Shaw BK, Kristan WB (1997) The neuronal basis of the behavioral choice between swimming and shortening in the leech: control is not selectively exercised at higher circuit levels. J Neurosci 17:786-795.

Soffe SR (1993) Two distinct rhythmic motor patterns are driven by common premotor and motor neurons in a simple vertebrate spinal cord. J Neurosci 13:4456-4469.

Thirumalai V, Prinz AA, Johnson CD, Marder E (2006) Red pigment concentrating hormone strongly enhances the strength of the feedback to the pyloric rhythm oscillator but has little effect on pyloric rhythm period. J Neurophysiol 95:1762-1770.

Wu JS, Due MR, Sasaki K, Proekt A, Jing J, Weiss KR (2007) State dependence of spike timing and neuronal function in a motor pattern generating network. J Neurosci 27:10818-10831.

Zelenin PV, Grillner S, Orlovsky GN, Deliagina TG (2001) Heterogeneity of the population of command neurons in the lamprey. J Neurosci 21:7793-7803.

Zhurov Y, Proekt A, Weiss KR, Brezina V (2005) Changes of internal state are expressed in coherent shifts of neuromuscular activity in Aplysia feeding behavior. J Neurosci 25:1268-1280. 